\title{
Hexahydroquinolines are antimalarial candidates with potent blood-stage and transmission-blocking activity
}

\author{
Manu Vanaerschot ${ }^{1}{ }^{1}$, Leonardo Lucantoni ${ }^{2}$, Tao Li $\mathbb{B}^{3}{ }^{3}$, Jill M. Combrinck ${ }^{4}$, Andrea Ruecker ${ }^{5,11,12}$, \\ T. R. Santha Kumar', Kelly Rubiano', Pedro E. Ferreira ${ }^{6}$, Giulia Siciliano7, Sonia Gulati', Philipp P. Henrich', \\ Caroline L. Ng', James M. Murithi' ${ }^{1}$, Victoria C. Corey ${ }^{8}$, Sandra Duffy ${ }^{2}$, Ori J. Lieberman ${ }^{1}$, M. Isabel Veiga ${ }^{6}$, \\ Robert E. Sinden ${ }^{5}$, Pietro Alano7, Michael J. Delves ${ }^{5}$, Kim Lee Sim ${ }^{3}$, Elizabeth A. Winzeler ${ }^{8}$, \\ Timothy J. Egan' ${ }^{\text {, Stephen L. Hoffman }}{ }^{3}$, Vicky M. Avery ${ }^{2}{ }^{2}$ and David A. Fidock ${ }^{1,10 \star}$
}

\begin{abstract}
Antimalarial compounds with dual therapeutic and transmission-blocking activity are desired as high-value partners for combination therapies. Here, we report the identification and characterization of hexahydroquinolines (HHQs) that show low nanomolar potency against both pathogenic and transmissible intra-erythrocytic forms of the malaria parasite Plasmodium falciparum. This activity translates into potent transmission-blocking potential, as shown by in vitro male gamete formation assays and reduced oocyst infection and prevalence in Anopheles mosquitoes. In vivo studies illustrated the ability of lead HHQs to suppress Plasmodium berghei blood-stage parasite proliferation. Resistance selection studies, confirmed by CRISPR-Cas9based gene editing, identified the digestive vacuole membrane-spanning transporter PfMDR1 ( $P$. falciparum multidrug resistance gene-1) as a determinant of parasite resistance to HHQs. Haemoglobin and haem fractionation assays suggest a mode of action that results in reduced haemozoin levels and might involve inhibition of host haemoglobin uptake into intra-erythrocytic parasites. Furthermore, parasites resistant to HHQs displayed increased susceptibility to several first-line antimalarial drugs, including lumefantrine, confirming that HHQs have a different mode of action to other antimalarials drugs for which PfMDR1 is known to confer resistance. This work evokes therapeutic strategies that combine opposing selective pressures on this parasite transporter as an approach to countering the emergence and transmission of multidrug-resistant $P$. falciparum malaria.
\end{abstract}

M alaria remains a global health calamity, with an estimated 212 million cases and 429,000 deaths ${ }^{1}$ in 2015, that occurs mostly in sub-Saharan Africa and results from infection with the Apicomplexan parasite Plasmodium falciparum (Pf). Improved malaria treatment and control has reduced morbidity and mortality worldwide by $40-50 \%$ since 2000 (ref. ${ }^{1}$ ), due largely to highly effective artemisinin-based combination therapies and mosquito vector control measures ${ }^{1,2}$. An important strategy to further advance the malaria elimination agenda is to include transmission-blocking agents in combination therapies ${ }^{3}$. Such agents inhibit the development of parasite gametocytes (GAMs), which represent an alternative form of intra-erythrocytic development to the pathogenic asexual blood stage (ABS) parasites and that mature over five stages 4 . Only stage V GAMS, which may survive days to weeks in circulation ${ }^{5}$, can continue the Pf lifecycle in Anopheles mosquitoes following ingestion of a parasitized blood meal. In the mosquito midgut, male GAMs exflagellate and produce microgametes that can fuse with female macrogametes to produce zygotes. Following subsequent development as ookinetes and oocysts, sporozoites are formed. Once inoculated in a new host, they will develop in the liver and initiate a new ABS cycle.

Primaquine is currently the only available drug with potent activity against stage $\mathrm{V} \mathrm{GAMs}{ }^{7,8}$, but it can be toxic for glucose6-phosphate dehydrogenase-deficient patients ${ }^{8}$. Hence, there is a compelling need to develop alternative transmission-blocking drugs. Recent advances in GAM-specific high-throughput screen technology $y^{9-19}$, combined with functional validation assays and drug action/resistance studies ${ }^{19-21}$, provide a powerful foundation to identify leads with Pf transmission-blocking activity.

In this study, we report a screen of the Novartis-GNF (The Genomics Institute of the Novartis Research Foundation) Malaria Box of ABS-active compound ${ }^{22}$ on Pf GAM stages and describe the identification and antimalarial properties of hexahydroquinolines (HHQs), a chemical class with considerable transmissionblocking potential.

'Department of Microbiology and Immunology, Columbia University Medical Center, New York, NY 10032, USA. '2Discovery Biology, Griffith Institute for Drug Discovery, Griffith University, Nathan 4111 Queensland, Australia. ${ }^{3}$ Sanaria Inc., Rockville, MD 20852, USA. ${ }^{4}$ Division of Pharmacology, Department of Medicine, University of Cape Town, Cape Town 7925, South Africa. ${ }^{5}$ Department of Life Sciences, Imperial College, London SW7 2AZ, UK. 6 Life and Health Sciences Research Institute (ICVS), School of Medicine, University of Minho, 4710-057 Braga, Portugal. ${ }^{7}$ Dipartimento di Malattie Infettive, Parassitarie ed Immunomediate, Istituto Superiore di Sanità, 00161 Rome, Italy. ${ }^{8}$ University of California, San Diego, School of Medicine, La Jolla, CA 92093, USA.

'Department of Chemistry, University of Cape Town, Cape Town 7700, South Africa. ${ }^{10}$ Division of Infectious Diseases, Department of Medicine, Columbia University Medical Center, New York, NY 10032, USA. "Mahidol-Oxford Tropical Medicine Research Unit, Faculty of Tropical Medicine, Mahidol University, Bangkok 10400, Thailand. Present address: ${ }^{12}$ Centre for Tropical Medicine and Global Health, Nuffield Department of Medicine, University of Oxford, Oxford OX3 7FZ, UK. *e-mail: df2260@cumc.columbia.edu 


\section{Results}

Identifying HHQs as potent gametocytocidal inhibitors. Using a luciferase (LUC)-based assay, we screened 3,825 compounds from the Novartis-GNF Malaria Box for activity against NF54 ${ }^{\text {Pfs } 16}$ early-stage GAMs. This screen yielded 797 compounds exhibiting $\geq 50 \%$ inhibition at $2.6 \mu \mathrm{M}$. Re-screening confirmed activity for 683 compounds, of which 176 showed $\geq 50 \%$ inhibition at $0.65 \mu \mathrm{M}$ (Fig. 1a). Screening the Novartis-GNF Malaria Box against late-stage GAMs using high-content imaging identified 572 hits with $\geq 50 \%$ inhibition at $2 \mu \mathrm{M}$ in at least two of the three replicates (Fig. 1a and Supplementary Fig. 1a).

Late-stage gametocytocidal hits were then prioritized for halfmaximum inhibitory concentration $\left(\mathrm{IC}_{50}\right)$ determination using both early- and late-stage GAM assays. Limited availability restricted the number of compounds to 149 (Supplementary Fig. 1b), yielding 71 and 60 compounds with $\mathrm{IC}_{50}$ values below $1 \mu \mathrm{M}$ for early- and latestage GAMs, respectively (Supplementary Table 1). Among these, 20 early-GAM and 16 late-GAM active compounds had $\mathrm{IC}_{50}$ values of $\leq 200 \mathrm{nM}$. In total, 43 compounds showed sub-micromolar activity against both stages (Fig. 1b). Reference drug controls showed expected values (Supplementary Table 2).

After deprioritizing chemical classes related to current antimalarial drugs or having liabilities likely to impede potential development, we identified four compounds with potent early- and late-stage gametocytocidal activity: GNF-Pf-5640, GNF-Pf-5660, GNF-Pf-5668 and GNF-Pf-5310 (Fig. 1c and Supplementary Table 3). These compounds also showed potent ABS activity $\left(\mathrm{IC}_{50}\right.$ values $<25 \mathrm{nM}$ ) against drug-sensitive 3D7 and multidrug-resistant Dd2 parasites, including a PfATP4 mutant line (Supplementary Fig. 2 and Supplementary Table 3). GNF-Pf-5640, GNF-Pf-5660 and GNF-Pf-5668 share a common scaffold belonging to the HHQ class of compounds (Fig. 1d), whereas GNF-Pf-5310 is a muscarinic-like compound (Supplementary Fig. 3). Cytotoxicity assays showed no inhibition at $40 \mu \mathrm{M}$ with GNF-Pf-5660, while the other three compounds caused $<50 \%$ inhibition at that concentration (Supplementary Fig. 2), indicating minimal toxicity against mammalian cells.

HHQs demonstrate potent in vitro transmission-blocking activity. Using an in vitro Pf dual gamete formation assay (DGFA), we investigated whether these four potent GAM-active compounds would also inhibit the development of cultured female and male gametes. The results indicated a potent inhibitory effect of the three HHQs on exflagellation when GAMs were exposed for $24 \mathrm{~h}$ and the compound carried over during the subsequent period of male gamete induction ('carry-over', Table 1). This effect persisted when compounds were washed out after $24 \mathrm{~h}$, that is, before gamete induction ('wash-out', Table 1). HHQs were thus effective against both mature male GAMs and developing male gametes, but showed no activity on female gamete formation. The muscarinic-like compound GNF-Pf-5310 showed no activity against either male or female gametes, contrasting with our transmission-blocking control methylene blue ${ }^{23,24}$ (Table 1).

GNF-Pf-5660, GNF-Pf-5668 and GNF-Pf-5310 were also tested in a membrane-feeding assay using Anopheles stephensi mosquitoes. This assay more closely mimics the natural parasite lifecycle than the DGFA, because compound-exposed Pf GAMs initiate the entire mosquito vector cycle from gametes to midgut oocysts. Pretreatment of GAMs with GNF-Pf-5660 or GNF-Pf-5668 at $3 \times$ their late-stage $\mathrm{IC}_{50}$ caused a $97 \%$ and $57 \%$ reduction, respectively, in the infection prevalence (that is, the percentage of infected mosquitoes, Fig. 2a), whereas the oocyst density per infected mosquito midgut was reduced by 95-99\% (Fig. 2b). GNF-Pf-5660- and GNF-Pf-5668exposed GAM cultures used for feeding to A. stephensi showed a $98 \%$ and $70 \%$ reduction, respectively, in their male GAM exflagellation rates (Fig. 2c), confirming earlier DGFA results. GNF-Pf-5310 was less active at similar exposure levels, causing no reduction in infection prevalence, a $73 \%$ reduction in oocyst density, and no effect on male GAM exflagellation rates (Fig. 2). As a result, GNF-Pf-5310 was deprioritized for further studies. The positive control methylene blue displayed maximal transmission-blocking activity (Fig. 2). Pre-treatment of GAMs with compounds at $1 \times$ their late-stage $\mathrm{IC}_{50}$ caused a lower to no effect on the parameters assessed (Fig. 2 and Supplementary Table 4a). The vehicle control DMSO showed no reductions compared to untreated controls.

In vivo activity of HHQs. HHQ antimalarial activity was assayed in Plasmodium berghei $(\mathrm{Pb})$-infected mice using the 4-day Peters test $^{25}$. Intraperitoneal injection of $70 \mathrm{mg} \mathrm{kg}^{-1}$ of GNF-Pf- 5660 or GNF-Pf-5668 reduced parasitaemias by $22 \%$ or $43 \%$, respectively, compared to vehicle-treated mice (Fig. 2d). At $100 \mathrm{mg} \mathrm{kg}^{-1}$, these agents reduced parasitaemia by $65 \%$ and $91 \%$, respectively (Fig. $2 \mathrm{~d}$ and Supplementary Table $4 \mathrm{~b}$ ). To assess the in vivo transmissionblocking activity of HHQs, infected mice were administered only two $100 \mathrm{mg} \mathrm{kg}^{-1}$ doses of GNF-Pf-5660 or GNF-Pf-5668. Following blood meals on GNF-Pf-5660-dosed mice, we observed a $25 \%$ and $21 \%$ reduction in the number of infected mosquitoes and their oocyst burdens, respectively (Fig. 2a,b). No effect was observed in the GNF-Pf-5668 mosquito cohort, although it displayed a $31 \%$ reduction in the number of male exflagellation centres at the time of mosquito feeding (Fig. 2c and Supplementary Table 4c). Limited in vivo transmission-blocking activity, as compared with our in vitro results, was also observed upon treating mice with two doses of dihydroartemisinin (DHA). Those assays showed 65\% and 75\% reductions in the number of infected mosquitoes and the oocyst burden, respectively, and found no reduction in the numbers of male exflagellation centres (Fig. 2a-c). These data are consistent with this two-dosing regimen having less impact on ABS parasitaemia than the 4 days of dosing used in the Peters test (Fig. 2d).

Resistance to HHQs is mediated by mutations in PfMDR1 and results in sensitization to clinical antimalarials. To gain insight into the mode of action of HHQs, we selected for Pf ABS resistance in vitro in the $\mathrm{Dd} 2-\mathrm{B} 2$ parasite line (the $\mathrm{B} 2$ clone of the $\mathrm{Dd} 2$ line), an approach that has proven effective for multiple antimalarial agents $^{26}$. Whole-genome sequence analysis of the resulting resistant clones identified the PfMDR1 (P. falciparum multidrug resistance gene-1) S1075I mutation in the GNF-Pf-5640-selected clone 2C4C3 and PfMDR1 Y290F in the GNF-Pf-5640-selected clone 3H9C7. These resistant clones showed a 77- and 79-fold increase in $\mathrm{IC}_{50}$ values for GNF-Pf-5640 compared to the parental Dd2-B2 line, respectively (Fig. 1e). The allele balance (AB) of the mutations in these clones was $50-59 \%$, consistent with their acquisition in one of the two pfmdr1 copies present in Dd2-B2. The GNF-Pf-5660-selected clone $2 \mathrm{~B} 6 \mathrm{H} 1$ and the GNF-Pf-5668-selected clone 3C4D2 showed $\mathrm{IC}_{50}$ increases of 248- and 37-fold, respectively, and had acquired the PfMDR1 Y290F (AB 100\%) or F1072L (AB 67\%) mutations, respectively (Fig. 1e). Shifts in $\mathrm{IC}_{90}$ values were similar to $\mathrm{IC}_{50}$ increases (Supplementary Tables 5 and 6).

Whole-genome sequencing data indicated that the GNF-Pf5668 -selected 3C4D2 clone also carried four copies of $p f m d r 1$, consistent with further gene amplification compared to the Dd2-B2 parent (which has two pfmdr1 copies in tandem). We were unable to determine whether the F1072L mutation (AB 67\%) was mainly located in the additional amplicons. Susceptibility testing of resistant clones indicated that each of the three observed PfMDR1 mutations conferred cross-resistance to all HHQs (Fig. 1e).

Because HHQ resistance was the highest in lines harbouring the Y290F mutation, we used a previously derived NF54 line ${ }^{27}$ that harboured this mutation $\left(\mathrm{NF} 54^{\text {selY290F }}\right.$ ) to verify whether Y290F also conferred resistance at the GAM stage. NF54 $4^{\text {selY290F }}$ early-stage GAMs showed a 40-74-fold $\mathrm{IC}_{50}$ shift for all HHQs compared to 
a

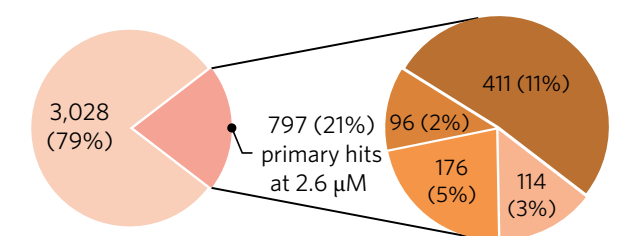

- Inactive $(50 \%$ at $>2.6 \mu \mathrm{M})$ - Unconfirmed $(50 \%$ at $>2.6 \mu \mathrm{M})$ $-50 \%$ at $\leq 0.65 \mu \mathrm{M}$

n $50 \%$ at $>1.3 \mu \mathrm{M}$ and $\leq 2.6 \mu \mathrm{M}$

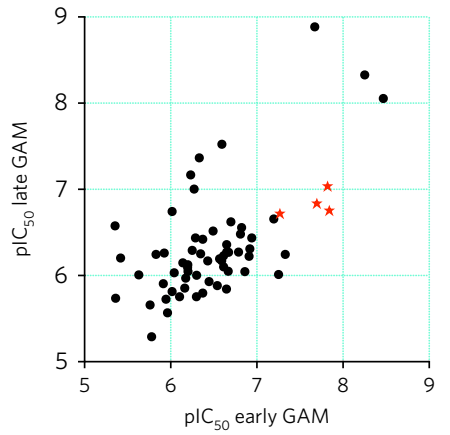

Late-stage gametocyte screen

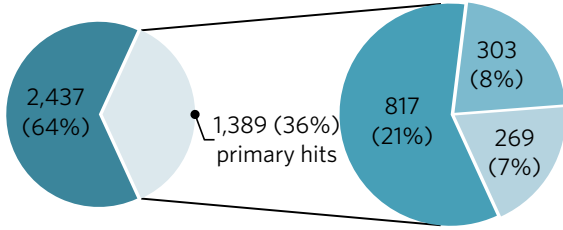

- Inactive (50\% at >2 $\mu \mathrm{M}) \quad 2$ replicates hit

$\square 1$ replicate hit $\quad 3$ replicates hit

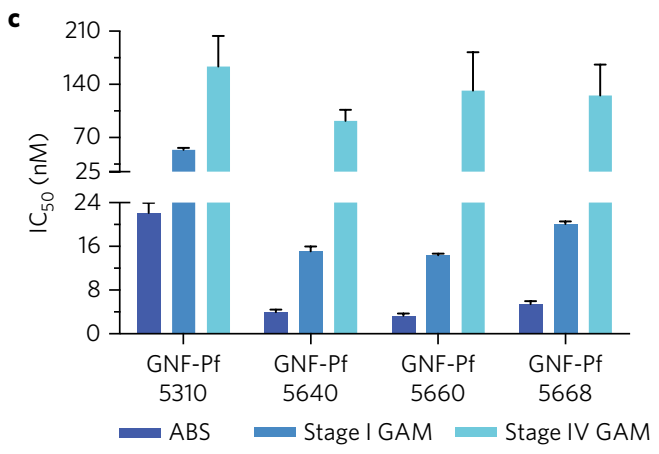<smiles>COc1ccc(C2CC(=O)C3=C(C2)NC(C)=C(C(=O)OC(C)C)C3c2ccccc2C)cc1OC</smiles>

GNF-Pf-5640<smiles>CCOC(=O)C1=C(C)NC2=C(C(C)CC(c3ccc(CC)c(CC)c3)C2)C1c1ccccc1</smiles>

GNF-Pf-5660<smiles>CCCOC(=O)C1=C(C)NC2=C(C(=O)CC(c3ccc(OC)c(OC)c3)C2)C1c1ccccc1F</smiles>

GNF-Pf-5668

e

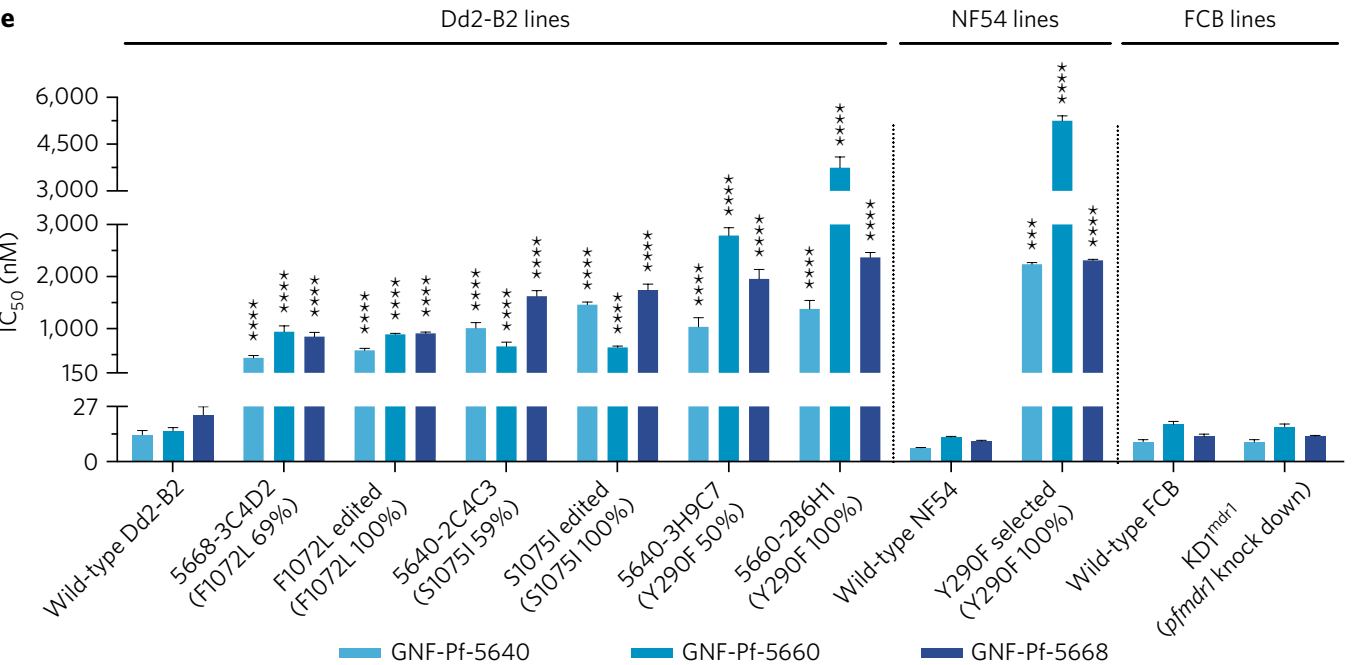

Fig. 1 | Identifying HHQs as potent Pf ABS and GAM inhibitors. a, Outcomes of the primary screening of the GNF-Novartis Malaria Box library against early- and late-stage Pf NF54 ${ }^{\text {Ps16 }}$ GAMs, using luciferase-based and high-content imaging assays, respectively. Compounds were classified based on the concentration at which $50 \%$ inhibitory activity was achieved. b. Scatterplot of early- and late-stage gametocytocidal potency of 63 compounds with dual activity (expressed as $\mathrm{pIC}_{50}$ values, which equal $-\log \left(\mathrm{IC}_{50}\right)$, where a value of 9 corresponds to $10^{-9} \mathrm{M}$, that is, $1 \mathrm{nM}$ ). Red stars correspond to the four compounds selected for the study. Data are based on two independent experiments, each probing both early- and late-stage GAMs. c, Intra-erythrocytic stage-specific activity of the four selected compounds was obtained from two independent experiments for each parasite stage (Supplementary Table 3 ). d, Structure of the three HHQs confirmed to have transmission-blocking activity. e, Impact of PfMDR1 mutations and copy number on HHQ potency. The $x$ axis depicts the individual mutant lines and their wild-type parental lines and indicates the GNF-Pf compound number, the clone number, and in parentheses the PfMDR1 mutation and its allele balance. 'Edited' implies that the Dd2-B2 line was edited using a CRISPR-Cas9 system to introduce the designated PfMDR1 mutation. Mean \pm s.e.m. $I_{50}$ values were calculated from three or more independent experiments (for details see Supplementary Table 5) and significance was assessed by a Welch's corrected $t$-test with Bonferroni-Dunn adjusted $P$ values for multiple comparisons when applicable: ${ }^{\star \star \star} P<0.001,{ }^{\star \star \star \star} P<0.0001$. 
Table 1 | Gamete formation inhibitory effect of the four selected compounds

\begin{tabular}{lllll} 
& \multicolumn{4}{c}{ Mean IC (lower 95\% Cl - upper 95\% Cl) } \\
\cline { 2 - 5 } & Male carry-over & Female carry-over & Male wash-out & Female wash-out \\
\hline GNF-Pf-5640 & $86.0 \mathrm{nM}(22.0-335.1)$ & $>20 \mu \mathrm{M}$ & $521.3 \mathrm{nM}(447.3-607.6)$ & $>20 \mu \mathrm{M}$ \\
GNF-Pf-5660 & $67.7 \mathrm{nM}(40.9-112.2)$ & $>20 \mu \mathrm{M}$ & $264.2 \mathrm{nM}(142.8-488.7)$ & $>20 \mu \mathrm{M}$ \\
GNF-Pf-5668 & $88.8 \mathrm{nM}(53.0-148.8)$ & $>20 \mu \mathrm{M}$ & $138.8 \mathrm{nM}(67.0-287.5)$ & $>20 \mu \mathrm{M}$ \\
GNF-Pf-5310 & $>20 \mu \mathrm{M}$ & $>20 \mu \mathrm{M}$ & $>20 \mu \mathrm{M}$ & $>20 \mu \mathrm{M}$ \\
Methylene blue & $61.5 \mathrm{nM}(38.8-97.3)$ & $161.8 \mathrm{nM}(111.4-234.9)$ & $146.7 \mathrm{nM}(116.3-185.1)$ & $846.2 \mathrm{nM}(490.6-1,460.0)$ \\
\hline
\end{tabular}

Compounds were carried over from GAM exposure to induction of gamete formation in the carry-over format of the assay, but were washed out before gamete formation in the wash-out format of the assay. $\mathrm{Cl}$ : $95 \%$ confidence interval based on four biological replicates.

wild-type NF54 (Supplementary Table 7). High levels of resistance were also observed at the late GAM stage, but because only very low numbers of NF54 $4^{\text {selY290F }}$ late GAMs could be obtained, accurate latestage $\mathrm{IC}_{50}$ values could not be calculated.

Structural modelling of PfMDR1 localized amino acid residue 290 to transmembrane domain (TMD) 5 and residues 1072 and 1075 to TMD 12. All three residues resided in the predicted drug-binding pocket cavity of PfMDR $1^{28}$, where they might mediate contact with substrates (Fig. 3a,b). Docking studies suggested that each mutation could significantly alter transporter interactions with HHQs. Interestingly, F1072L and Y290F seemed to decrease the minimum estimated energy required for docking (Supplementary Tables 8-12 and Supplementary Fig. 4). This effect was less pronounced for S1075I, which could be due to this residue not being fully exposed to the drug-binding pocket.

To test whether these point mutations in PfMDR1 were causal for resistance, we implemented a pfmdr1-specific CRISPR-Cas9 strategy $^{27}$. Transfection of parental Dd2-B2 parasites yielded the PfMDR1 $1^{\text {edF1072L }}$ and PfMDR1 $1^{\text {eds1075I }}$ clones that expressed the F1072L and S1075I mutations, respectively, observed in both $p f m d r 1$ copies. These clones showed an essentially equivalent gain of resistance to HHQs as the original drug-pressured lines, confirming the causal role of the PfMDR1 mutations in mediating resistance (Fig. 1e). Despite repeated efforts, we were unable to introduce the Y290F mutation into the parental line.

Testing KD $1^{\text {mdr1 }}$, a pfmdr1 knockdown line previously generated in FCB parasites, showed that a decreased $p f m d r 1$ copy number did not affect HHQ susceptibility (Fig. 1e). Similarly, increased $p f m d r 1$ copy number also did not noticeably affect HHQ susceptibility (Fig. 1e), as shown by the similar $\mathrm{IC}_{50}$ values of GNF-Pf-5668-3C4D2 (harbouring an estimated four copies of $p f m d r 1$ in addition to the F1072L mutation) and PfMDR1 $1^{\text {edF1072L }}$ (with the F1072L mutation engineered into the wild-type $\mathrm{Dd} 2-\mathrm{B} 2$ line with two $p f m d r 1$ copies).

The impact of the three PfMDR1 mutations on Pf ABS susceptibility to known antimalarials was determined using PfMDR1 $1^{\text {edF1072L, }}$ PfMDR1 ${ }^{\text {edS1075I }}$ and GNF-Pf-5660-2B6H1 (also referred to as PfMDR1 ${ }^{\text {selY290F }}$ (Fig. 3c). For all three lines, no changes in susceptibility were observed for piperaquine, chloroquine (CQ), the active metabolite monodesethyl-CQ (md-CQ), md-amodiaquine (md-ADQ) or ferroquine (Fig. 3 and Supplementary Tables 13 and 14). In contrast, PfMDR $1^{\text {edF1072L }}$ and PfMDR $1^{\text {selY290F }}$ showed a higher susceptibility to both quinine (QN) (3.6- and 3.7-fold, respectively) and mefloquine (MQ) (3.6- and 2.0-fold, respectively), whereas PfMDR1 ${ }^{\text {eds1075I }}$ showed a 5.1-fold increased QN susceptibility but only a 1.4 -fold increased susceptibility to MQ. Lumefantrine (LMF) showed a similar trend, with PfMDR edF1072L $^{\text {en }}$ and PfMDR $1^{\text {selY290F }}$ showing a higher LMF susceptibility (3.1- and 1.9-fold, respectively), whereas PfMDR ${ }^{\text {edS1075I }}$ remained unchanged. All three mutations significantly increased Pf susceptibility to DHA by $\sim 1.6$-fold. Parasite susceptibility to the experimental ACT451840 compound, a known inhibitor of PfMDR1 ${ }^{27}$, was decreased by 16-, 5.5- and 45-fold for PfMDR1 $1^{\text {edF1072L }}$, PfMDR1 $1^{\text {edS1075I }}$ and PfMDR1 ${ }^{\text {selY290F, }}$, respectively (Fig. 3c).

To assess the effects of $p f m d r 1$ copy number variation in combination with the F1072L mutation, we compared the antimalarial susceptibilities of GNF-Pf-5668-3C4D2 (harbouring four pfmdr1 copies $+\mathrm{F} 1072 \mathrm{~L}(\mathrm{AB} 69 \%)$ ) to that of PfMDR1 ${ }^{\text {edF1072L }}$ (with two pfmdr1 copies +F1072L (AB 100\%)). The results indicated a decreased susceptibility to QN, MQ and LMF (that is, higher $\mathrm{IC}_{50}$ values) in GNF-Pf-5668-3C4D2 compared to PfMDR1 ${ }^{\text {edF1072L }}$ (Fig. 3c). Importantly, these $p f m d r 1$ copy number-induced changes in GNF-Pf-5668-3C4D2 did not fully compensate for the effects introduced by the F1072L mutation, as this line still showed a increased susceptibility to QN (1.7-fold) and MQ (1.4-fold) compared to the wild-type Dd2-B2 control (Fig. 3c). These data provide evidence that the F1072L mutation had a major effect on increasing parasite susceptibility to QN, MQ and LMF that was only partially reversed by increases in $p f m d r 1$ copy number.

HHQs inhibit haemoglobin endocytosis. Previous studies have shown that mutant PfMDR1 isoforms can modulate the degree of Pf ABS resistance to several antimalarial drugs, including CQ and $\mathrm{ADQ}$, in lines expressing mutant $p f c r t^{29-31}$. These 4-aminoquinolines are known to inhibit haemozoin $(\mathrm{Hz})$ formation and cause a buildup in free haem ${ }^{32,33}$. In light of the finding that PfMDR1 confers Pf resistance to HHQs, we performed subcellular haem fractionation assays to assess whether these agents might also affect $\mathrm{Hz}$ formation in ABS parasites (Fig. 4 and Supplementary Table 15).

In parental Dd2-B2 parasites, we observed that haemoglobin $(\mathrm{Hb})$ and free haem concentrations per parasite remained unchanged across all GNF-Pf-5660 exposure levels (up to $2 \times \mathrm{IC}_{50}$ ) (Fig. 4). Hz levels, however, showed a 1.5 -fold decrease when parasites were exposed to as little as $0.1 \times$ the GNF-Pf-5660 $\mathrm{IC}_{50}$ value, as compared with untreated controls. A 2.3 -fold reduction in $\mathrm{Hz}$ levels was observed at higher exposure levels $\left(0.25 \times\right.$ to $2 \times \mathrm{IC}_{50}$, Fig. 4). Total haem iron levels also showed similar reductions with increasing concentrations of GNF-Pf-5660 (Fig. 4). A similar profile of reduced $\mathrm{Hz}$ and total haem iron content per parasite was also

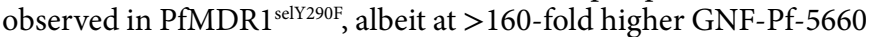
exposure levels than in Dd2-B2, consistent with the fold increase in $\mathrm{IC}_{50}$ in the mutant line (Fig. 4).

For comparison, we also assessed the haem fractionation profiles of MQ and LMF. In the Dd2-B2 line, MQ and LMF showed little or no effect compared to untreated controls, with the exception of a 1.6-fold decrease in $\mathrm{Hz}$ levels in parasites exposed to $2 \times$ the MQ $\mathrm{IC}_{50}$. Intriguingly, PfMDR $1^{\text {selY290F }}$ showed MQ profiles similar to those observed with GNF-Pf-5660, that is, reductions in $\mathrm{Hz}$ and total haem iron levels with increasing MQ concentrations. In PfMDR 1 selY290F, LMF also caused a reduction in $\mathrm{Hz}$ and total haem iron amounts at $1 \times$ to $2 \times \mathrm{IC}_{50}$ concentrations (Fig. 4 ).

We also measured the amount of $\mathrm{Hb}$ endocytosed by late rings exposed to GNF-Pf-5660, WR99210, MQ or LMF, using western 


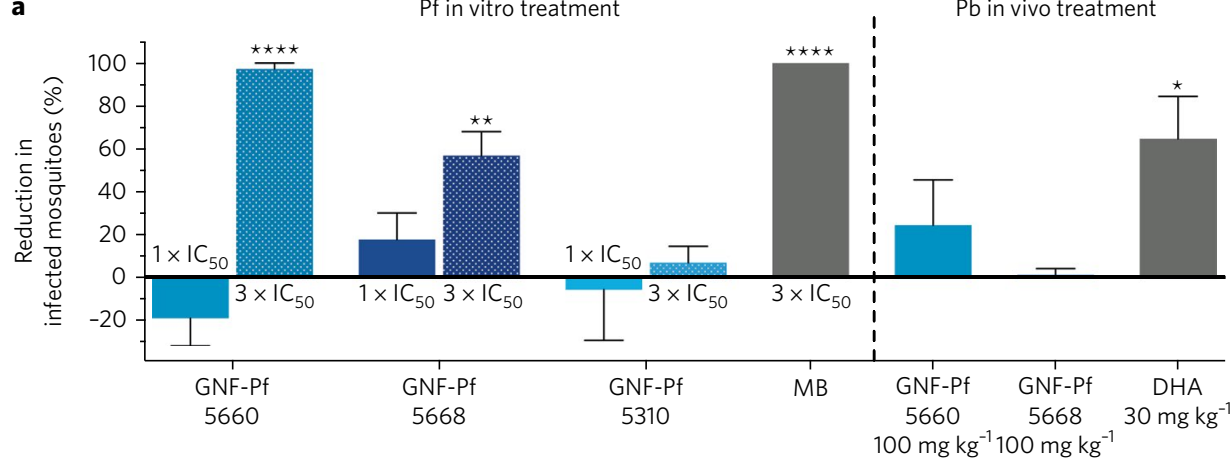

b

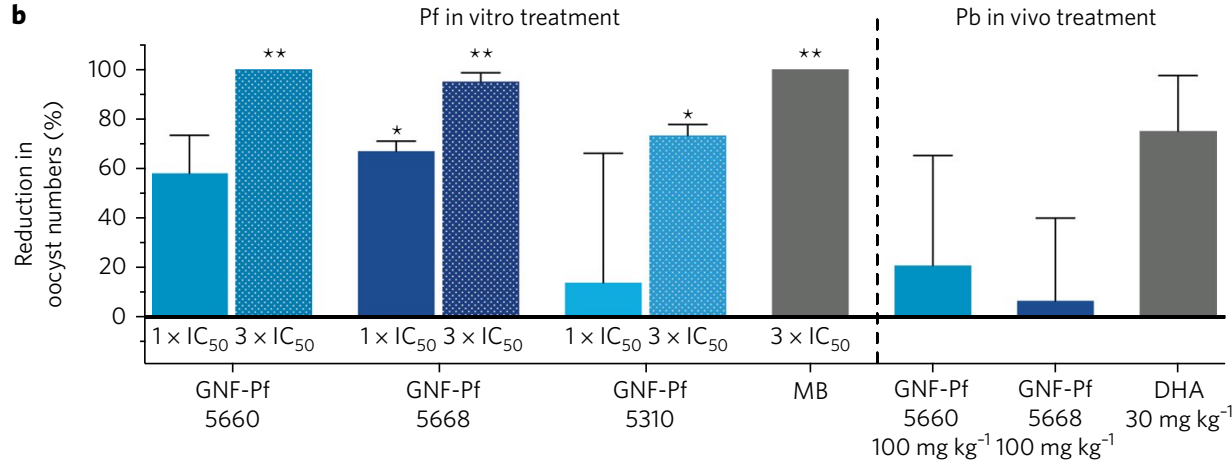

c
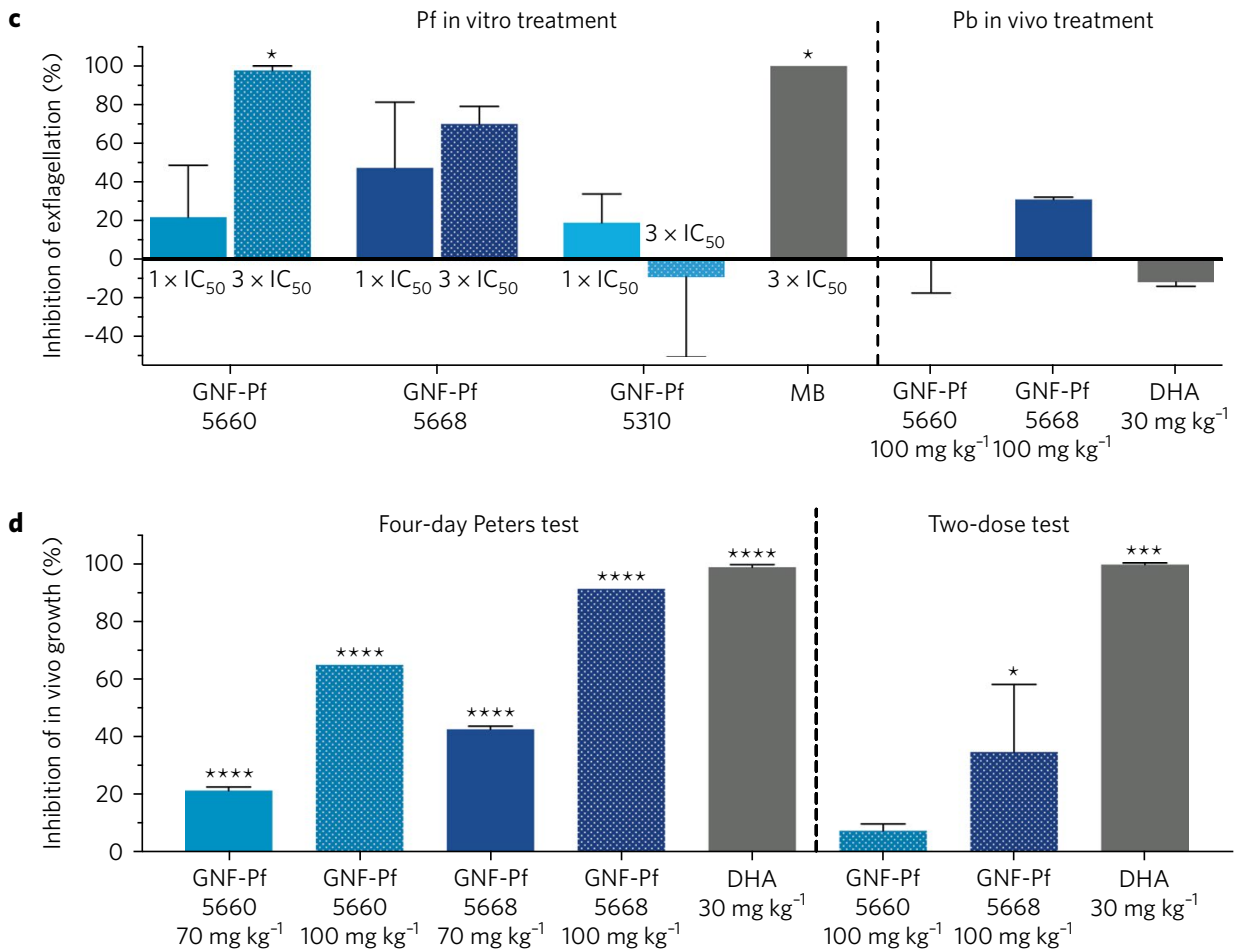

Fig. 2 | HHQs demonstrate potent transmission-blocking activity and inhibit male gamete exflagellation. For in vitro treatment studies, mosquitoes were fed with compound or DMSO-treated Pf GAMs. Compounds were added at $1 \times$ or $3 \times$ their late-stage GAM IC ${ }_{50}$ and the DMSO vehicle was used as a negative control. Parasites were treated for 3 days, and drug was then removed for 4 days before the blood meal feeds and transmission-blocking studies. For in vivo studies, mosquitoes were fed on Pb-infected mice that were treated with two doses of $100 \mathrm{mg} \mathrm{kg}{ }^{-1}$ of the $\mathrm{GNF}$ compounds, or solvent as a negative control, or $30 \mathrm{mg} \mathrm{kg}^{-1} \mathrm{DHA}$ as a positive control. $\mathbf{a}$, Per cent reduction in number of infected mosquitoes. $\mathbf{b}$, Per cent reduction in number of oocysts per midgut. c, Compound effect on male GAM exflagellation, expressed as the average number of exflagellation centres per field in GAM cultures. Results in a-c are shown as mean \pm s.e.m., calculated from two independent experiments (see Methods). d, Mean per cent reduction of bloodstage parasites in $\mathrm{Pb}$-infected mice treated with $\mathrm{HHQs}$, or DHA as a positive control. Data are shown as mean \pm s.e.m. based on two independent repeats, except for the $100 \mathrm{mg} \mathrm{kg}^{-1} \mathrm{HHQ}$-treated mice in the 4-day Peters test that were tested once and served as a pilot experiment for the two-dose test (to match the two-dose design used in the transmission-blocking studies shown in a-c). Statistical significance was assessed using a Welch's corrected $t$-test with Bonferroni-Dunn adjusted $P$ values for multiple comparisons when applicable: ${ }^{\star} P<0.05,{ }^{\star \star} P<0.01,{ }^{\star \star \star} P<0.001,{ }^{\star \star \star \star} P<0.0001$. All data are provided in Supplementary Tables 4a-c. MB, methylene blue. 


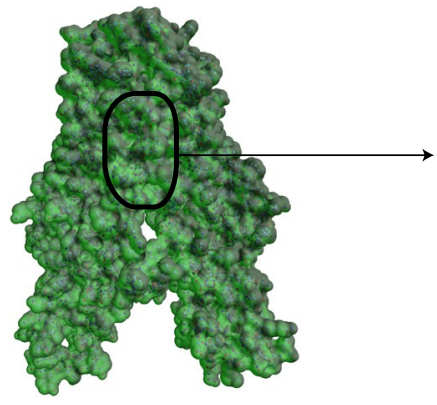

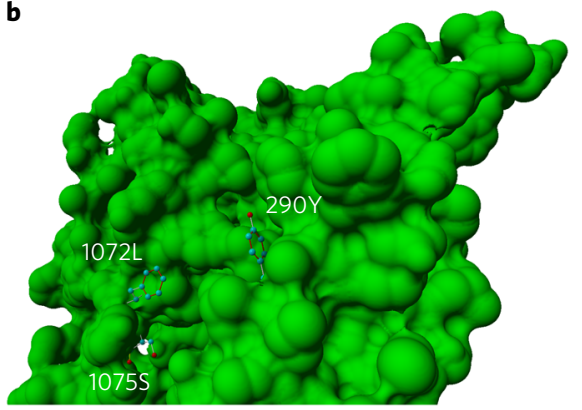
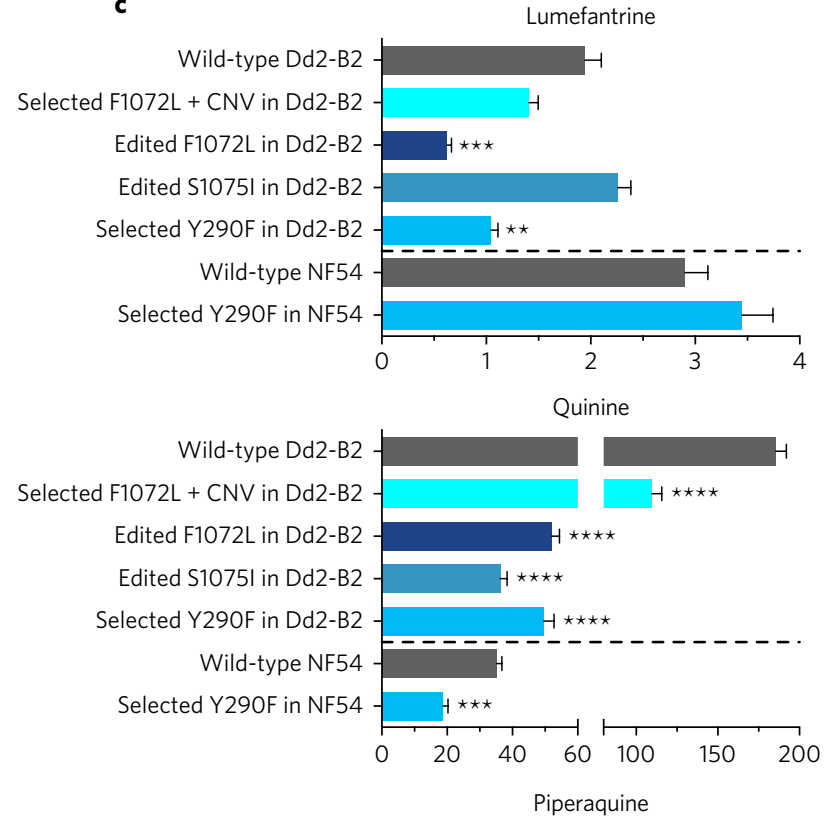

Wild-type Dd2-B2

Selected F1072L + CNV in Dd2-B2

Edited F1072L in Dd2-B2

Edited S1075I in Dd2-B2

Selected Y290F in Dd2-B2

Wild-type NF54

Selected Y290F in NF54

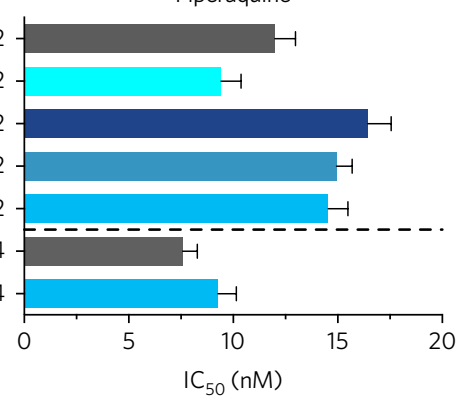

Mefloquine
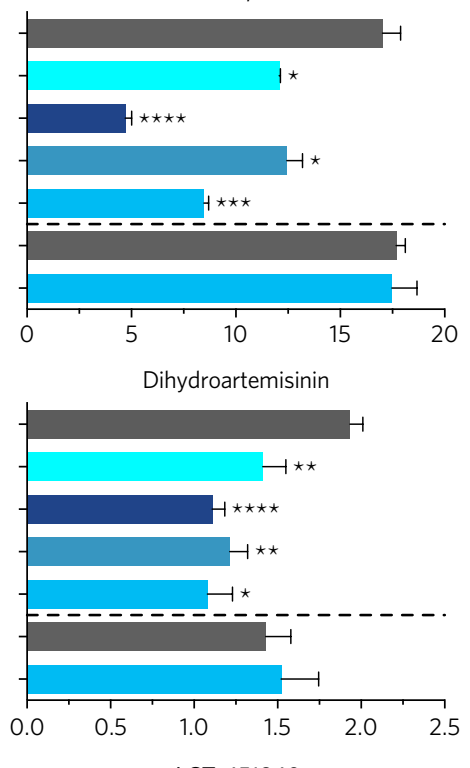

ACT -451840

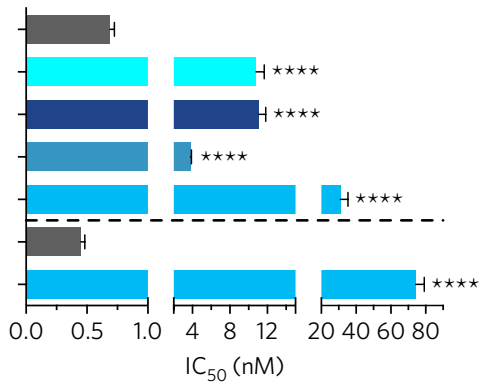

Fig. 3 | PfMDR1 mutations associated with HHQ resistance localize to its drug-binding pocket and can sensitize parasites to first-line antimalarial drugs and the preclinical candidate ACT-451840. a, Structural model of PfMDR1 transmembrane domains (TMDs) based on the 4Q4H structure. b. Visualization of the drug-binding pocket cavity. TMD 7 and TMD 8 were omitted to allow visualization of the mutations. c, Susceptibility of PfMDR1 mutants, obtained through HHQ selection or genetic editing, to other antimalarials. Mean \pm s.e.m. IC $\mathrm{C}_{50}$ values were calculated from 3 to 12 independent experiments (Supplementary Table 13). IC $C_{90}$ data followed similar trends as the $I C_{50}$ data described herein (Supplementary Table 14). Statistical significance was assessed using a Welch's corrected $t$-test with Bonferroni-Dunn adjusted $P$ values for multiple comparisons when applicable: ${ }^{\star} P<0.05$, ${ }^{\star \star} P<0.01,{ }^{\star \star \star} P<0.001,{ }^{\star \star \star \star} P<0.0001 . C N V$, copy number variation.

blot analysis of wild-type Dd2-B2 or HHQ-resistant PfMDR1 ${ }^{\text {selY290F }}$ parasites (Fig. 5 and Supplementary Table 16). Results showed that the $\mathrm{Hb}$ levels within parasites were reduced by $32 \%$ in GNF-Pf5660-exposed Dd2-B2, but remained unchanged in PfMDR $1^{\text {selY290F. }}$ $\mathrm{Dd} 2-\mathrm{B} 2$ parasites showed no significant changes in $\mathrm{Hb}$ levels when exposed to WR99210, MQ or LMF.

\section{Discussion}

Here, we describe the antimalarial properties of HHQs, a class of potent gametocytocidal antimalarials with ABS activity that were identified by screening the Novartis-GNF Malaria Box against early- and late-stage Pf GAMs. Functional assays with Pf GAMs and gametes confirmed potent transmission-blocking activity. Moderate activity with our initial hits was also observed in vivo in $\mathrm{Pb}$ mouse models. Mode of action and resistance studies implicated a key role for the digestive vacuole (DV) transporter PfMDR1. We also provide evidence that $\mathrm{HHQ}$ s reduce levels of $\mathrm{Hz}$ and total haem iron in treated parasites and inhibit the initial uptake of $\mathrm{Hb}$ from host red blood cells (RBCs). Cross-resistance assays also revealed that HHQ-resistant parasites became sensitized to several antimalarials 
in current clinical use, emphasizing the potential added value of HHQs for combination treatments.

The confirmed hit rates of $18 \%$ and $15 \%$ obtained from our compound screens of early- and late-stage GAMs, respectively
(Fig. 1a), highlight the value of screening ABS-active compound libraries as a source for identifying gametocytocidal compounds. The limited overlap (20\%) between early- and late-stage GAM hits (Supplementary Figure 1b) suggests that GAMs at different
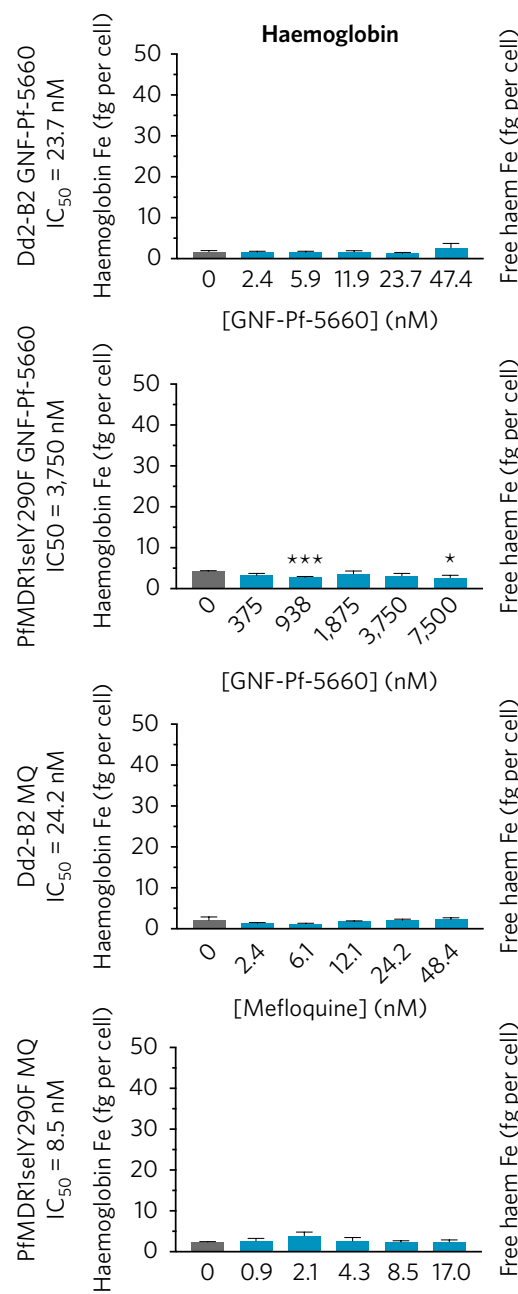

[Mefloquine] (nM)
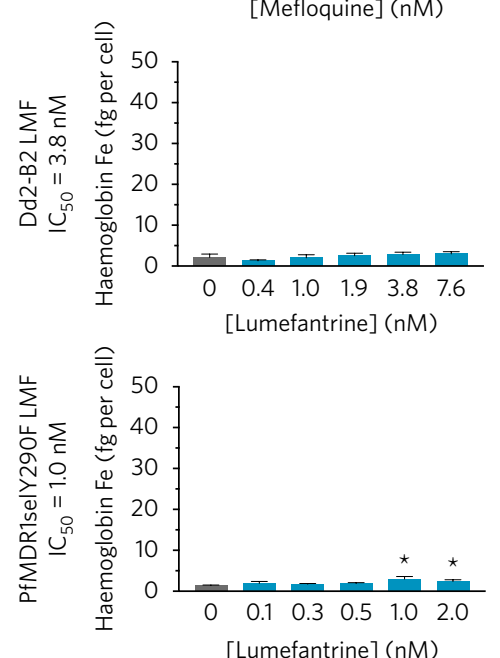
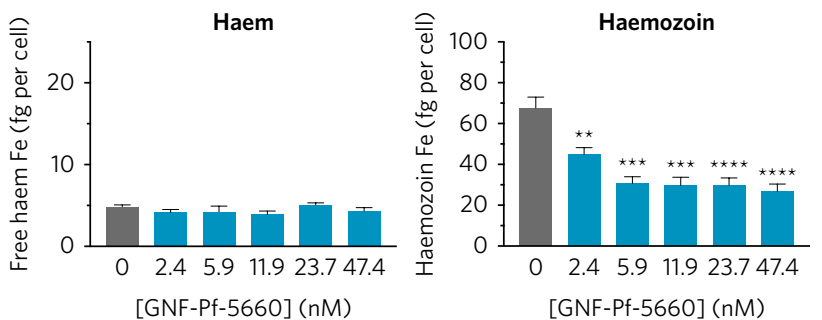

[GNF-Pf-5660] (nM)

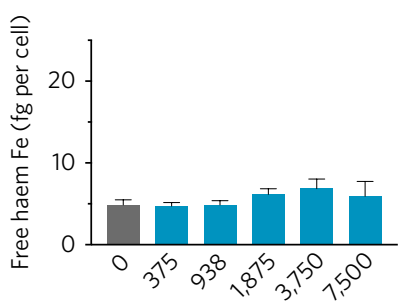

[GNF-Pf-5660] (nM)
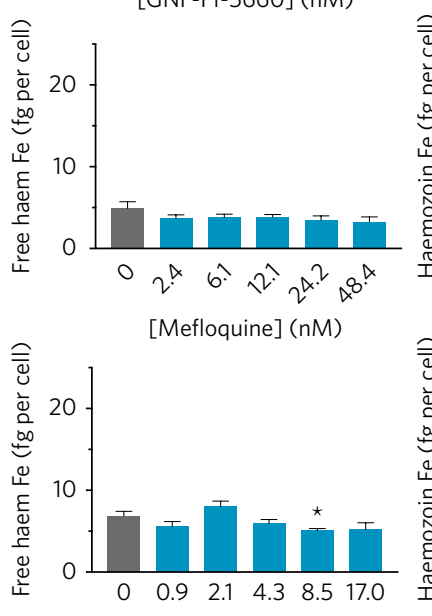

[Mefloquine] (nM)
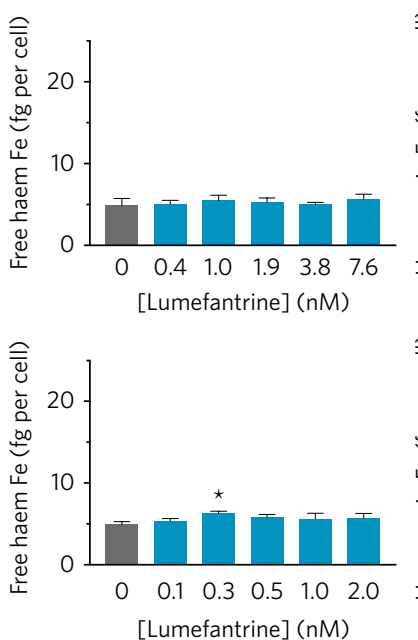

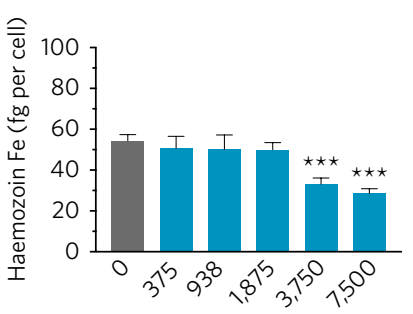

[GNF-Pf-5660] (nM)
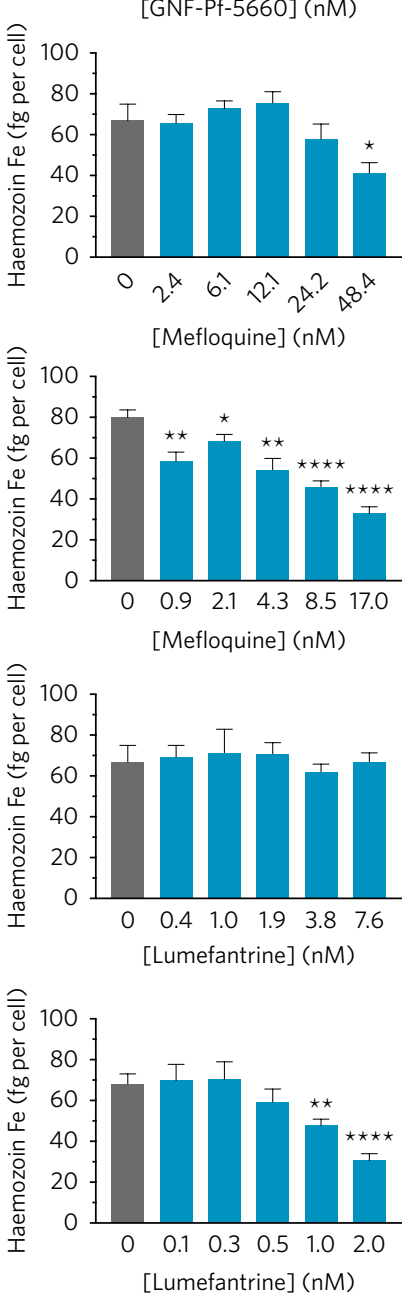
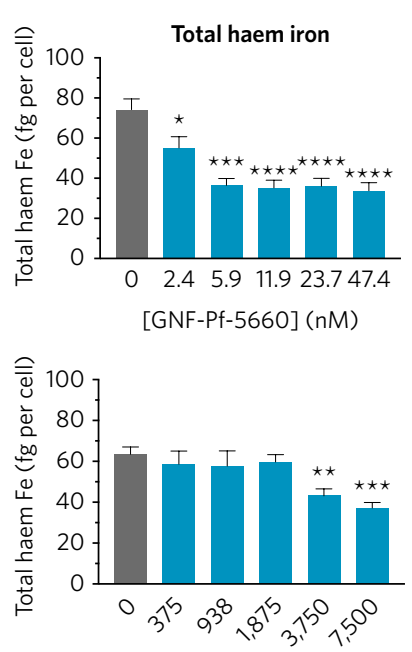

[GNF-Pf-5660] (nM)

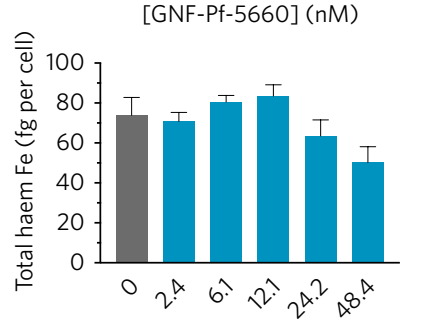

[Mefloquine] (nM)
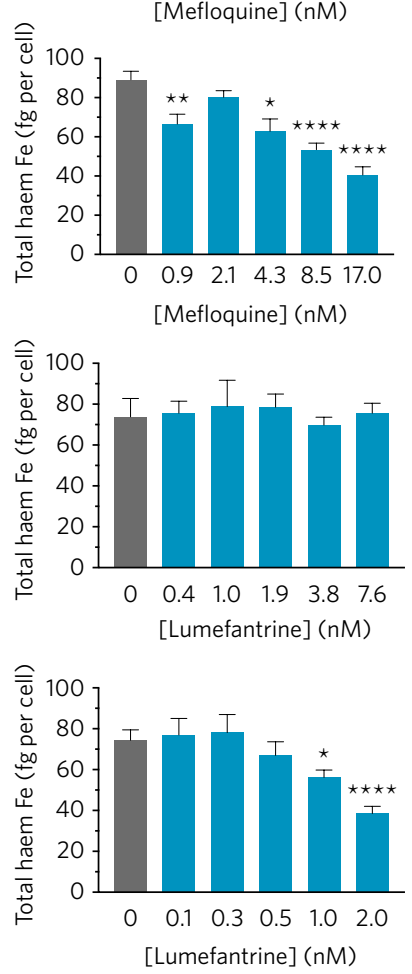

Fig. 4 | GNF-Pf-5660 causes a decrease in Hz and total haem iron levels in wild-type ABS Dd2-B2 and mutant HHQ-resistant PfMDR1 ${ }^{\text {selY290F }}$ parasites, the latter at significantly higher compound concentrations. Synchronized parasites were exposed to GNF-Pf-5660 or the reference antimalarials MQ and $\mathrm{LMF}$ and fractionated to measure $\mathrm{Hb}$, free haem, or $\mathrm{Hz}$ iron. Total haem iron represents the sum of these three values. Results show $\mathrm{Hb}$, free haem (which could be loosely associated with lipids or other biomolecules), $\mathrm{Hz}$ and total haem iron levels (as $\mathrm{fg}$ per cell) in parasites exposed to a range of $\mathrm{IC}_{50}$ values $(0.1,0.25,0.5,1.0$ and 2.0x). Mean \pm s.e.m. amounts were calculated from two independent experiments and statistical significance was assessed by a two-tailed $t$-test: ${ }^{\star} P<0.05,{ }^{\star \star} P<0.01,{ }^{\star \star \star} P<0.001,{ }^{\star \star \star \star} P<0.0001$. All data are provided in Supplementary Table 15. MQ, mefloquine; $L M F$, lumefantrine. 
developmental stages express distinct sets of druggable targets, provided that technology-related differences such as incubation times, sensitivity or specific bias for selecting certain chemical classes were minimal in both screens.

Our HHQ hits GNF-Pf-5640, GNF-Pf-5660 and GNF-Pf-5668 showed potent activity against ABS parasites as well as early- and latestage GAMs (Fig. 1c), with only minimal toxicity against mammalian cells. These compounds were inactive against cultured $\mathrm{Pb}$ liverstage parasites ${ }^{16}$, possibly because $\mathrm{Hb}$ import and metabolism does not occur in parasitized hepatocytes.

The results provided herein also document potent in vitro HHQ activity on male GAMs and gametes, which translated into marked transmission-blocking activity in mosquitoes (Table 1 and Fig. 2). In Pb-infected mice, HHQs showed moderate efficacy: the 4-day Peters tests indicated a potent inhibition of ABS growth at $100 \mathrm{mg} \mathrm{kg}^{-1}$, whereas the two-dose assay showed only a limited impact on ABS parasitaemia and parasite transmission (Fig. 2). The high doses required in vivo, compared to the lower doses at which activity was observed in vitro, suggest that HHQs might be rapidly metabolized or otherwise inactivated or eliminated by the host. The ester group present in HHQs, for instance, is probably sensitive to host esterases ${ }^{34}$. These HHQs are the first hits from this class and structure-activity relationship studies and chemical optimization will be required. Enhancing HHQ transmission-blocking activity in vivo is an important goal, in light of the limited efficacy observed to date (Fig. 2). We note that earlier studies of $\mathrm{Pb}$ reported that a $30 \%$ reduction in oocyst numbers per infected mosquito sufficed to eliminate the parasite from a murine population after several transmission cycles $^{35}$. In light of these data, HHQs constitute a promising class of compounds with transmission-blocking potential that merit pharmacokinetic and pharmacodynamic optimization.

In vitro HHQ resistance selection studies identified three mutations in PfMDR1 (Y290F, F1072L and S1075I), which were confirmed by CRISRP/Cas9 editing to be causal for ABS resistance (Fig. 1e). PfMDR1-associated resistance also extended to the GAM stage (Supplementary Table 7). We note that the Pf3k collection of over 2,500 Pf genomes collected worldwide (www.malariagen. net/apps/pf3k) contained no mutations with an $\mathrm{AB}>0.2$ at these PfMDR1 positions, suggesting that they are very rare, if not nonexistent, in the field.

Structural modelling studies indicated that all three amino acids co-localize in the predicted drug-binding pocket of PfMDR1 (Fig. 3) and generally caused a closer interaction between HHQs and mutant PfMDR1 (Supplementary Tables 8-12). Further studies are required to determine the nature of the interactions between HHQs and wild-type versus mutant PfMDR1 and how this relates to HHQ potency.

PfMDR1, an ATP-binding cassette (ABC) transporter, can mediate the transport of several antimalarial drugs across the DV membrane ${ }^{30,36,37}$. In the highly acidic DV compartment, Pf proteases degrade $\mathrm{Hb}$ from the host $\mathrm{RBC}$, providing a source of the amino acids required for protein synthesis and parasite growth ${ }^{38}$. This catabolic process triggers the formation of highly reactive haem moieties, which Plasmodium normally detoxifies through biomineralization into chemically inert $\mathrm{Hz}$ crystals in the DV. Several antimalarials act either by binding toxic haem species and inhibiting $\mathrm{Hz}$ formation (CQ or ADQ), or in the case of artemisinins by apparently being activated through interaction with haem iron ${ }^{39}$. Mutations in PfMDR1, or the chloroquine resistance transporter PfCRT that also resides on the DV membrane, are known to affect in vitro parasite susceptibility to these drugs, presumably by affecting drug transport, and are associated with an increased risk of parasite recrudescence or treatment failure in patients $\mathrm{s}^{30,40-42}$.

Haem fractionation studies showed a different profile in parasites treated with GNF-Pf-5660 as compared to $\mathrm{CQ}^{32}, \mathrm{ADQ}^{32}, \mathrm{MQ}$ or LMF (Fig. 4), indicating a distinct mode of action. HHQs did not

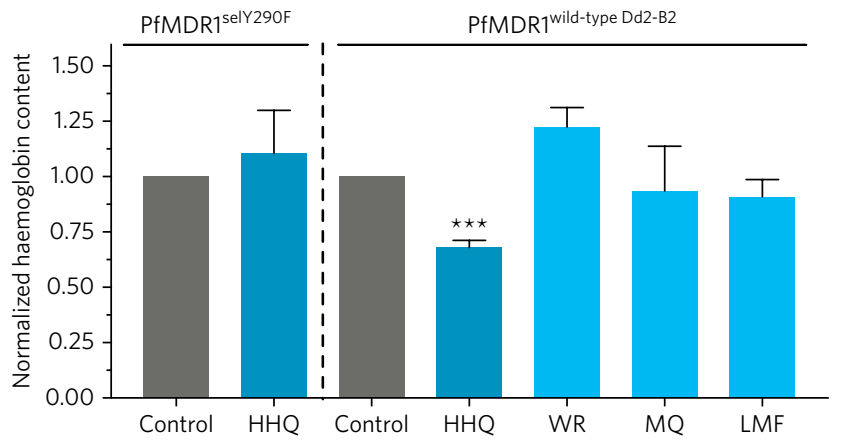

Fig. 5 | HHQs inhibit $\mathbf{H b}$ endocytosis. Parasites were exposed to $5 \times$ the $I_{50}$ of the HHQ GNF-Pf-5660 or reference antimalarials, and Hb content was measured by western blot analysis, with amounts normalized to mock DMSO-treated control parasites. Mean \pm s.e.m. values were calculated from three to four independent experiments. Statistical significance was assessed by a Welch's corrected $t$-test adjusted for multiple comparisons with the Bonferroni-Dunn method: ${ }^{\star \star \star} P<0.001$. HHQ, GNF-Pf-5660; WR, WR99210; MQ, mefloquine; LMF, lumefantrine; CQ, chloroquine. All data are provided in Supplementary Table 16 and Supplementary Fig. 5a,b.

directly inhibit haem conversion into $\mathrm{Hz}$, but were found to reduce the total amounts of intracellular haem iron. These data suggest that HHQs act upstream of the $\mathrm{Hb}$ digestion pathway by interfering with parasite-mediated uptake of host $\mathrm{Hb}$, which delivers the substrate for $\mathrm{Hz}$ formation. Western blot data showed a reduction of total $\mathrm{Hb}$ levels in HHQ-treated wild-type Dd2-B2 but not HHQ-resistant PfMDR1 ${ }^{\text {selY290F }}$ parasites (Fig. 5), supporting an effect of HHQs on parasite-mediated $\mathrm{Hb}$ endocytosis.

Parasite lines resistant to CQ, pyrimethamine and PfATP4 inhibitors remained sensitive to HHQs (Supplementary Table 3), whereas HHQ-resistant lines showed an increased Pf ABS susceptibility to QN, MQ, DHA and LMF (except for PfMDR1 ${ }^{\text {edS1075I }}$, which showed no difference for LMF). Susceptibility to CQ, md-CQ, md-ADQ, ferroquine and piperaquine remained unaltered in HHQ-resistant parasites (Fig. 3 and Supplementary Table 13). QN, MQ, DHA and LMF are thought to interact at some level with haem but also act outside of the DV, raising the possibility that they might engage with the $\mathrm{Hb}$ import and catabolism process that begins with endocyto$\operatorname{sis}^{33,43-45}$. We speculate that the PfMDR1 mutations causing HHQ resistance might sensitize parasites to $\mathrm{MQ}$ and $\mathrm{LMF}$ by preventing their sequestration by wild-type PfMDR1 in the DV, away from their primary site of action ${ }^{46}$.

HHQ-resistant ABS parasites showed no substantial change in their susceptibility to the haem-binding drugs CQ or md-ADQ that act primarily in the $\mathrm{DV}^{41}$. Interestingly, PfMDR1 Y290F was also recently identified in selections using ACT-451840, a piperazinebased pre-clinical candidate with low nanomolar activity against Pf ABS parasites and GAMs ${ }^{27}$. Studies with the ACT-213615 analogue earlier showed direct binding to PfMDR1, identifying it as a potential target ${ }^{47}$. The unusually high levels of resistance to both HHQs and ACT- 451840 conferred by each of the three mutations (Figs 1e and $3 \mathrm{c}$ ) suggest that both compound series probably share a similar mode of action. These data also evoke the possibility that PfMDR1 might itself be a target of HHQs, a hypothesis that can be investigated using HHQ analogues to study protein interactions with PfMDR $1^{47}$ in cultured parasites or heterologous expression systems ${ }^{48}$.

Stage-specific profiling showed that $p f m d r 1$ is maximally transcribed around the time of merozoite egress (www.PlasmoDB.org), suggesting that PfMDR1 is already present in early- to mid-stage rings, well before ABS parasites have formed their mature DV. This timing, supported by western blot data ${ }^{49}$, suggests that PfMDR1 might be involved in vesicle-mediated endocytosis of host $\mathrm{Hb}$ from 
the RBC en route to the developing DV. Such a role for PfMDR1 would be consistent with our observations that HHQ inhibition reduces intra-parasitic total haem iron and $\mathrm{Hz}$ levels in trophozoites and reduces $\mathrm{Hb}$ levels in late-stage rings (Figs 4 and 5).

Further studies into the stage-dependency and subcellular site of action of HHQs and of drugs that are impacted by HHQ-selected mutations in PfMDR1, such as the first-line agent LMF, should yield interesting insights into how these agents act and how to leverage this information into identifying promising drug combinations. Ideally, such combinations would exert opposing selective pressures on mutant and wild-type PfMDR1, in an effort to stall the emergence and spread of multidrug resistance. This approach is currently being used in clinical trials where artemether + LMF is being combined with ADQ (Clinical Trial NCT02453308), based on earlier reports that LMF and ADQ exert opposing selective pressures on wild-type and mutant forms of both $p f m d r 1$ and $p f c r t^{40,50}$. In addition, the potent activity of HHQs against both ABS parasites and GAMs highlights the promise of developing this chemical class into a partner for future antimalarial combination therapies with transmission-blocking activity.

\section{Methods}

Parasite cultures and strains. Pf ABS parasites were cultured at 3-4\% haematocrit (Hct) in human $\mathrm{O}^{+}$RBCs in RPMI-1640 media, supplemented with $25 \mathrm{mM}$ HEPES, $50 \mu \mathrm{M}$ hypoxanthine, $2 \mathrm{mM} \mathrm{L}$-glutamine, $2 \mathrm{gl}^{-1}$ sodium bicarbonate, $0.5 \%$ (wt/vol) AlbuMAXII (Invitrogen) and $10 \mu \mathrm{g} \mathrm{ml}^{-1}$ gentamycin, in $5 \% \mathrm{O}_{2}$, $5 \% \mathrm{CO}_{2}$ and $90 \% \mathrm{~N}_{2}$ at $37^{\circ} \mathrm{C}$. For GAM cultures, Albumax was replaced with $10 \%$ human serum. 3D7 and Dd2 parasites were originally obtained from T. Wellems (NIAID, NIH). Dd2-B2 is a genetically homogenous parasite line that was cloned from Dd2 by limiting dilution. The NITD609-resistant parasites (generated in the Dd2 background) and the NF54 ${ }^{\text {Pfs16 }}$ line have been reported previously ${ }^{23,51}$. The NF54 $4^{\text {selY290F }}$ and FCB pfmdr1 knockdown line $\left(\mathrm{KD}^{\mathrm{mdr} 1}\right)$ and their corresponding wild-type lines were obtained in two earlier studies ${ }^{27,52}$. A list of all parasite lines generated in this study is provided in Supplementary Table 17. Parasite lines were screened by PCR for Mycoplasma every 2-3 months.

Compound screening against gametocytes. The Novartis-GNF Malaria Box consisted of 3,825 known inhibitors of ABS Pf parasites with a median $\mathrm{IC}_{50}$ of $0.53 \mu \mathrm{M}$ (25th percentile, $0.23 \mu \mathrm{M}$; 75th percentile, $1.09 \mu \mathrm{M})$, as determined by a SYBR Green-based $72 \mathrm{~h}$ growth inhibition assay ${ }^{53}$, and was obtained as $10 \mu \mathrm{l}$ of $1 \mathrm{mM}$ stock solution in 100\% DMSO. These compounds were independently screened against early- and late-stage GAMs (stages I and IV, respectively), as described below in the two subsequent sections. Screening was performed with the NF54 $4^{\mathrm{Pfs} 16}$ line that expresses a green fluorescent protein (GFP)-LUC fusion under the $p f s 16$ promoter that is active throughout gametocytogenesis ${ }^{23}$ Earlier studies have reported the optimization of high-throughput early- and late-stage GAM screens using this line in LUC- or GFP-based assays ${ }^{5-56}$. For the LUC-based screens with early-stage GAMs, compounds were diluted 1.5-fold in $100 \%$ DMSO and further diluted in water to a final assay concentration of $2.6 \mu \mathrm{M}$ in $0.4 \%$ DMSO. Hits, defined as compounds that showed $\geq 50 \%$ inhibition at $2.6 \mu \mathrm{M}$, were retested for confirmation at $2.6,1.3$ and $0.65 \mu \mathrm{M}$. For the GFP high-content imaging-based screens with late-stage GAMs, compounds were diluted to a final assay concentration of $2 \mu \mathrm{M}$ in $0.4 \%$ DMSO and screened in triplicate. Due to limitations in the availability of the screening hits, not all hits could be retested for confirmation. Fresh batches of 125 hits from the late-stage screens were obtained as $2 \mathrm{mM} 100 \%$ DMSO stock solutions from GNF-Novartis, and 24 additional hits were purchased as solids from various commercial sources (Supplementary Table 1)

Luciferase-based early-stage gametocyte inhibition assay. LUC-based screening with stage I GAMs was conducted as previously described ${ }^{13}$. Briefly, NF54 ${ }^{\text {Pfsit }}$ parasites were synchronized by two cycles of $5 \%$ sorbitol treatment, and gametocytogenesis was induced by applying multiple known stimulant stress factors (a drop in Hct, higher parasitaemia and spent media). After one replication cycle under these conditions, the resulting stage I GAMs and asexual rings were separated from residual $\mathrm{Hz}$-containing and later-stage GAMs by paramagnetic purification over a MACS column (Miltenyi Biotech). Medium containing $50 \mathrm{mM}$ $N$-acetyl-D-glucosamine (to eliminate ABS parasites) was provided and refreshed daily. On day 1 of gametocytogenesis, which corresponds to day 0 of the early-stage GAM assay, the parasitaemia was adjusted to $10 \%$ and the Hct was reduced to $0.5 \%$. Parasites were seeded under sterile conditions to a volume of $45 \mu \mathrm{l}$ per well in 384-well white luminescence plates (CulturPlate, PerkinElmer) using a Multidrop dispenser (Thermo Scientific). Experimental compounds were added to the plates at the desired concentrations in a volume of $5 \mu \mathrm{l}$ with a final DMSO concentration of $0.4 \%$ per assay well. Two columns of each plate were used as in-plate controls, containing 0.4\% DMSO (negative control, $0 \%$ inhibition) or $5 \mu \mathrm{M}$ puromycin (positive control, $100 \%$ inhibition), respectively. Plates were sealed with gas-exchange membranes (Breathe-easy, Sigma) and incubated for $72 \mathrm{~h}$ at $37^{\circ} \mathrm{C}$ in $5 \% \mathrm{O}_{2}, 5 \% \mathrm{CO}_{2}$ and $90 \% \mathrm{~N}_{2}$. On day 4 of gametocytogenesis, $25 \mu \mathrm{l}$ of supernatant was removed from each well and replaced with $15 \mu$ l Steadylite solution (luciferase assay kit, PerkinElmer), using a Biomek FX (Beckman Coulter) high-throughput liquid handling system. Plates were sealed and incubated at room temperature for $60 \mathrm{~min}$ to allow for complete cell lysis, after which the plates were read using a MicroBeta Trilux luminometer (PerkinElmer).

Image-based late-stage gametocyte inhibition assay. The effect of compounds on stage IV GAMs was determined using an image-based assay ${ }^{54}$. Briefly, at day 8 post-induction, stage IV NF54 $4^{\text {Pfs16 }}$ GAMs were purified over a MACS column and adjusted to $10 \%$ parasitaemia and $0.1 \%$ Hct. Parasites were dispensed in $45 \mu \mathrm{l}$ volumes into 384 -well imaging plates containing $5 \mu \mathrm{l}$ of diluted compounds per well, with a final DMSO concentration of $0.4 \%$. After $72 \mathrm{~h}$ incubation at $37^{\circ} \mathrm{C}$ in $5 \% \mathrm{O}_{2}, 5 \% \mathrm{CO}_{2}$ and $90 \% \mathrm{~N}_{2}, 5 \mu \mathrm{l}$ of $0.07 \mu \mathrm{g} \mathrm{ml}^{-1}$ MitoTracker Red CM- $\mathrm{H}_{2}$ XRos (Invitrogen) in PBS was added and incubated overnight. Parasites were imaged on an Opera QEHS confocal imaging system (PerkinElmer). Image analysis using an Acapella-based script determined the number of viable mature GAMs per imaged area of each well, as described previously ${ }^{54}$. Controls included $5 \mu \mathrm{M}$ puromycin. Experiments were performed with triplicate technical replicates on three independent occasions.

Gametocytocidal potency. Stage-specific potency data were generated using LUC and imaging-based assays for early- and late-stage GAMs, respectively, as described above. Relevant parasite stages were exposed to serial dilutions of compounds, typically with a maximal concentration of $40 \mu \mathrm{M}$, in 14 to 21 serial dilution steps with three concentrations per log dose. Assays were repeated on two independent occasions. Reference compounds (puromycin, DHA, CQ, pyrimethamine and primaquine) were used as positive and negative controls and yielded $\mathrm{IC}_{50}$ values (Supplementary Table 2) that were consistent with earlier reports ${ }^{13,55}$

Imaging-based ABS high-throughput assay. Hit compounds with confirmed gametocytocidal activity were also evaluated for ABS activity using our imagingbased high-throughput assay ${ }^{57}$. Briefly, sorbitol-synchronized Pf parasite lines were seeded at $2 \%$ parasitaemia and $0.3 \%$ Hct in 384-well clear-bottom imaging plates (CellCarrier, PerkinElmer). For this work we used 3D7 (drug-sensitive), $\mathrm{Dd} 2$ (resistant to CQ, MQ and pyrimethamine) and NITD609- $\mathrm{R}^{\mathrm{Dd} 2}$ clone \#2 (resistant to the PfATP4 inhibitor now termed KAE609 ${ }^{51}$ ). Parasites were incubated with compounds for $72 \mathrm{~h}$ at $37^{\circ} \mathrm{C}$ in $5 \% \mathrm{O}_{2}, 5 \% \mathrm{CO}_{2}$ and $90 \% \mathrm{~N}_{2}$. Parasites were then permeabilized using $0.01 \%$ Triton-X100 and $0.001 \%$ saponin plus $5 \mathrm{mM}$ EDTA and stained using $0.5 \mu \mathrm{g} \mathrm{ml}^{-1} 4^{\prime}, 6$-diamidino-2-phenylindole (DAPI). Plates were kept at room temperature in the dark for a minimum of $6 \mathrm{~h}$ and were then imaged on an Opera confocal microplate system (PerkinElmer) using a 20x water objective with $405 \mathrm{~nm}$ excitation and $420-490 \mathrm{~nm}$ bandpass emission filter and an exposure time of $80 \mathrm{~ms}$. The experiment was performed with two independent cultures. Parasite numbers, calculated based on spot size, intensity and fluorescence over background, were determined using Acapella software ${ }^{57}$.

HEK293 cytotoxicity. Cytotoxicity of the selected gametocytocidal hits was assessed against the HEK293 human embryonic kidney cell line (ATCC \#CRL-1573), maintained in DMEM medium (Life Technologies) supplemented with $10 \%$ fetal calf serum (Gibco). Cells were trypsinized, seeded into sterile 384 -well plates (Falcon) at 2,000 cells per well in $45 \mu \mathrm{l}$ medium and incubated overnight at $37^{\circ} \mathrm{C}$ in $5 \% \mathrm{CO}_{2}$ and $95 \%$ humidity to allow adhesion. A resazurin-based metabolic assay was used to determine cell viability ${ }^{58}$. Briefly, test compounds were serially diluted and added to the plates to generate a dose-response with a maximal concentration of $40 \mu \mathrm{M}$, in wells containing a final DMSO concentration of $0.4 \%$. Plates were incubated for $72 \mathrm{~h}$ under standard conditions, after which the medium was removed and replaced with $35 \mu \mathrm{l}$ of serum-free DMEM containing $44 \mu \mathrm{M}$ resazurin. The plates were then incubated for a further $4-6 \mathrm{~h}$ under standard conditions before being read on an EnVision plate reader (PerkinElmer) using $530 \mathrm{~nm} / 595 \mathrm{~nm}$ fluorescence excitation/emission settings. Two biological replicates were performed. The cells were certified by ATCC as being Mycoplasma-free at source.

Dual gamete formation assay. The effects of GNF-Pf-5310, GNF-Pf-5640, GNF-Pf-5660 and GNF-Pf-5668 on the formation of male and female gametes were determined using the DGFA ${ }^{56}$. Compounds were tested across a range of 12 concentrations $(0.84 \mathrm{nM}$ to $20 \mu \mathrm{M})$ and $\mathrm{IC}_{50}$ values were calculated using nonlinear regression (Graphpad Prism 6). Briefly, stage V GAMs were exposed to compounds for $24 \mathrm{~h}$, after which gamete formation was triggered in the presence (carry-over format) or absence (wash-out format, three washes) of compound. Male gamete formation was assessed by automated microscopic detection of exflagellation and female gamete formation was evaluated by live immunostaining for Pfs 25 surface expression ${ }^{59}$. Data were calculated from four biological replicates, with methylene blue included as a control. 
Membrane-feeding assay. Late-stage GAMs (12 days post GAM induction) were treated for 3 days with GNF-Pf-5660, GNF-Pf-5668 or GNF-Pf-5310 at $1 \times$ or $3 \times$ their late-stage $\mathrm{GAM} \mathrm{IC}_{50}$ values (with daily medium changes including compound refreshment) and then maintained without compound for another 4 days. Methylene blue was used as a positive control at a concentration of $3 \times$ the $\mathrm{GAM} \mathrm{IC}_{50}$. GAMs treated with $0.25 \%$ DMSO were used as the negative control ${ }^{23}$. Stage V GAM cultures at $50 \% \mathrm{Hct}$ and $0.01-0.16 \%$ parasitaemia were exposed to A. stephensi mosquitoes (4-6 days post-emergence) for $30 \mathrm{~min}$ using an artificial membrane feeding apparatus. For each compound and controls, two containers holding 25 to 35 parasite-exposed mosquitoes each were maintained at $26^{\circ} \mathrm{C}$, $78 \%$ relative humidity with a $12 \mathrm{~h}$ day/night cycle. Seven or eight days post feeding (for experiments 1 and 2, respectively), the midguts from blood-fed female mosquitoes ( $n=17-21$ per container) were dissected and stained with $0.2 \%$ mercurochrome to count oocyst numbers. Infection prevalence was defined as the percentage of mosquitoes harbouring one or more midgut oocysts, and infection intensity was defined as the mean number of oocysts per mosquito. Midguts of mosquitoes fed with DMSO vehicle-treated GAMs showed an average of 19 oocysts. All samples were blinded for both dissection and oocyst counting.

In vivo potency of HHQs on ABS parasites. For our 4-day Peters test, four female CD-1 mice (Taconic; 5 weeks old, weighing 22-25g) per group were infected intraperitoneally with $1 \times 10^{6} \mathrm{P}$. berghei ANKA MRA868 ABS parasites. Treatments with 70 or $100 \mathrm{mg} \mathrm{kg}^{-1}$ of GNF-Pf-5660 or GNF-Pf-5668, $30 \mathrm{mg} \mathrm{kg}^{-1}$ DHA or solvent-only (20\% DMSO in water) were administered intraperitoneally $2 \mathrm{~h}, 24 \mathrm{~h}, 48 \mathrm{~h}$ and $72 \mathrm{~h}$ after infection. Blood-stage parasitaemias were assessed at $96 \mathrm{~h}$ post infection by microscopy. Plasma compound concentrations were not measured. All experiments involving mice were reviewed and approved by the Columbia University Institutional Animal Care and Use Committee. Sample size was determined in accordance with published literature $e^{60,61}$ and to minimize the number of animals under experiment. All research animals were randomly allocated into treated and control groups and groups were blinded for collection and analysis of data. This research is compliant with federal regulations detailed in the Animal Welfare Act (AWA) (7 U.S.C. 2131, seq.) and the Health Research Extension Act of 1985 (Public Law 99-158).

In vivo transmission-blocking effect of HHQs. To assess the in vivo transmission-blocking effect of HHQs, four female CD-1 mice were treated with two doses of $100 \mathrm{mg} \mathrm{kg}^{-1}$ GNF-Pf-5660 or GNF-Pf-5668, $30 \mathrm{mg} \mathrm{kg}^{-1} \mathrm{DHA}$, or solvent only. Doses were spaced $12 \mathrm{~h}$ apart, starting when blood-stage parasitaemia after infection with $5 \times 10^{6} \mathrm{P}$. berghei ANKA MRA868 parasites reached $1 \%$, to measure drug impact only on GAMs. We note that in P. berghei GAMs develop and become transmissible within a $24 \mathrm{~h}$ period. Twelve hours after the last dose, both blood-stage parasitaemia and the level of inhibition of male GAM exflagellation were assessed. For the exflagellation test, a tail blood sample was collected in a heparinized tube, mixed with ookinete medium (RMPI medium, $25 \mathrm{mM}$ HEPES, $50 \mathrm{mgl}^{-1}$ hypoxanthine, $2 \mathrm{gl}^{-1}$ sodium bicarbonate and $0.5 \%$ Albumax) containing $100 \mu \mathrm{M}$ xanthurenic acid and evaluated by microscopy after a $10 \mathrm{~min}$ incubation at $20^{\circ} \mathrm{C}$. Shortly afterwards, 25-30 A. stephensi mosquitoes were fed on individual mice that were anaesthetized by intraperitoneal injection of $200 \mu \mathrm{l}$ of a mixture of ketamine $\left(17.5 \mathrm{mg} \mathrm{ml}^{-1}\right)$ and xylazine $\left(2.5 \mathrm{mg} \mathrm{ml}^{-1}\right)$. Unfed mosquitoes were removed, and engorged mosquitoes were fed daily with a $10 \%$ sugar solution and maintained at $20^{\circ} \mathrm{C}$ at $80 \%$ humidity for 10 days. Midguts of $10-20$ mosquitoes fed per mouse were then dissected and stained with $0.5 \%$ mercurochrome for oocyst counting by microscopy. Data were analysed in a similar manner as for the membrane-feeding assay (see section 'Membrane-feeding assay').

Selections for HHQ resistance. To select for Pf resistance to GNF-Pf5640, GNF-Pf-5660 and GNF-Pf-5668, triplicate flasks of $10^{9} \mathrm{Dd} 2-\mathrm{B} 2$ parasites were exposed to compounds at $5 \times$ their $\mathrm{ABS} \mathrm{IC}_{50}$ values. Briefly, medium containing the compound of interest was refreshed daily during the first 7 days, while cultures were carefully monitored to avoid overgrowth and ensure that sensitive parasites were effectively cleared. RBCs $(0.5 \% \mathrm{Hct})$ were added on day 7 and the total culture volume was reduced by $25 \%$ each subsequent week until a $5 \mathrm{ml}$ volume was obtained. Fresh RBCs (1\% Hct) were added on a weekly basis from day 14 onwards, always maintaining a total Hct level of 3-4\%. Once cultures appeared negative, the medium containing the compound of interest was refreshed every other day until at least $1 \%$ parasitaemia was reached, after which medium was replenished daily. Resistant clones were obtained by limiting dilution ${ }^{62}$. DNA was extracted with the DNAeasy Blood \& Tissue kit (Qiagen) and sequenced using paired-end reads on a Illumina HiSeq 2500 platform ${ }^{27}$. Whole-genome sequence analysis was performed at UC San Diego and Columbia University, using previously described customized pipelines and the GATK toolkit ${ }^{26,63,64}$.

Gene editing. $p f m d r 1$ point mutations were introduced into Dd2-B2 parasites using a two-plasmid CRISPR-Cas9 targeted gene-editing strategy ${ }^{27}$. The Cas9 plasmid encoded the Cas 9 nuclease, a single guide RNA (5'-GAAAATTAAT GTCCTTAAA for F1072L and S1075I and either 5' -ATTTTAGTTTCT TATGCATT or $5^{\prime}$-TTACATATAGGTTTAATAAA for Y290F) that complexes the Cas 9 nuclease with the target sequence, and a human $d h f r$ cassette that mediates resistance to the WR99210 selection agent ${ }^{27}$. The donor plasmid contained the homologous pfmdr1 sequence (nucleotides 1871-3421 of the Pf3D7_0523000 coding sequence for the mutations at amino acid position 1072 and 1075, and nucleotides 383-1034 for the mutation at amino acid position 290) harbouring the mutation of interest, as well as eight synonymous mutations to prevent the Cas9 nuclease from cleaving the edited gene sequence, and the blasticidin S deaminase gene cassette that mediates resistance to blasticidin. Ring-stage cultures were transfected with the Cas9 and donor plasmids as described in ref. ${ }^{27}$. At $24 \mathrm{~h}$ after electroporation, blasticidin $\left(2 \mu \mathrm{g} \mathrm{ml}^{-1}\right)$ and WR99210 (2.5 nM) selection was initiated. The medium containing these drugs was refreshed daily for 12 days. From day 13 onwards, cultures were maintained under blasticidin pressure until parasites were microscopically observed. pfmdr1-modified clones were obtained by limiting dilution, and gene editing was verified by PCR and Sanger sequencing. Transfections were performed in duplicate on one to three occasions until editing was achieved.

\section{Drug susceptibility profiling of HHQ-resistant lines in ABS parasites. To} assess antimalarial susceptibilities, we exposed ring-stage cultures (at an initial parasitaemia of $0.2 \%$ and $1 \% \mathrm{Hct}$ ) for $72 \mathrm{~h}$ to a range of ten concentrations (plated in duplicate), in a 96-well format. Dd2-B2 parasites were included as a parental control. Parasite survival was assessed by flow cytometry on an Accuri C6 (BD Biosciences) using SYBR Green and MitoTracker Deep Red FM (Life Technologies) as nuclear and vital dyes ${ }^{65}$. Assays were performed on three to ten separate occasions (Supplementary Table 5). Growth inhibition data were plotted using nonlinear regression to calculate $\mathrm{IC}_{50}$ values (using Graphpad Prism 6). $\mathrm{IC}_{50}$ values were compared between the selected or edited lines and their parental controls (Dd2-B2, FCB or NF54 $4^{\text {Pfs16 }}$ ) using an unequal variance $t$-test, also referred to as Welch's $t$-test, to correct for differences in the number of repeats per group. A Bonferroni-Dunn correction for multiple comparisons was performed when applicable.

Drug susceptibility of HHQ-resistant lines at GAM stages. The NF54 $4^{\mathrm{selY} 290 \mathrm{~F}}$ line was tested together with a wild-type NF54 parental control for GAM susceptibility to the three HHQs using a previously described parasite lactate dehydrogenasebased assay ${ }^{6}$.

PfMDR1 modelling. The Pf 3D7 PfMDR1 amino acid sequence (accession no. XP_001351787) was used to develop a structural model. Membrane protein topology was predicted using TOPCONS ${ }^{67}$. Residues 51-350 and 781-1090, based on the topology prediction, were used for homology detection and structure prediction with the HHpred tool ${ }^{68}$. The best ranked hits $(\mathrm{Prob}=100)$ were considered and the ABC transporter structure of Thermotoga maritima (PDB 4Q4H) was used to model PfMDR1, using MODELLER because of its apo state ${ }^{69}$. PfMDR1 TMDs were refined using WinCoot ${ }^{70}$ and assembled by superimposition on the $4 \mathrm{Q} 4 \mathrm{H}$ template using the visualization software Yasara ${ }^{71}$. Docking simulations of compounds GNF-Pf-5640, GNF-Pf-5660 and GNF-Pf-5668 were performed using DockingServer ${ }^{72}$, with residues 290, 1072 and 1075 seeded in a $15 \AA$ box at default conditions.

Haem fractionation assay. Haem fractionation analysis was performed as described in ref. ${ }^{32}$. Briefly, early ring-stage sorbitol-synchronized Dd2-B2 and PfMDR1 $1^{\text {selY290F }}$ parasites were exposed to GNF-Pf-5660, MQ or LMF at concentrations corresponding to $0.1,0.25,0.5,1$ and $2 \times$ the $\mathrm{ABS}_{\mathrm{IC}_{50}}\left(\right.$ ref. $^{32}$ ). After a $32 \mathrm{~h}$ incubation, cells were saponin-lysed and trophozoites were subjected to a series of lysis, solubilization and differential centrifugation steps to obtain fractions that contain $\mathrm{Hb}$, free haem or $\mathrm{Hz}$. The UV-visible spectra of haem as $\mathrm{Fe}(\mathrm{III})$ haem-pyridine were analysed in each of these fractions and compared to a haem standard curve for accurate quantification. These quantities were subsequently normalized using the number of analysed cells, as determined by flow cytometry of an aliquot of exposed cells. Two-tailed $t$-tests were used to assess significance ${ }^{32}$

Western blotting. Sorbitol-synchronized parasites were exposed to $5 \times$ the $\mathrm{IC}_{50}$ of GNF-Pf-5660, WR99210, MQ or LMF for $5 \mathrm{~h}$, starting at the late-ring stage, that is, before parasites entered the mid-stage trophozoites that have maximal rates of $\mathrm{Hb}$ degradation and $\mathrm{Hz}$ formation. $\mathrm{RBCs}$ were then saponin-lysed and parasites washed five times in $1 \times$ PBS. The parasite pellet was solubilized in SDS-PAGE sample buffer and sonicated for ten cycles of $10 \mathrm{~s}$. Samples were then boiled for $10 \mathrm{~min}$ at $90^{\circ} \mathrm{C}$ and $3.5 \times 10^{6}$ parasites were loaded on a $4-20 \%$ Criterion TGX precast midi protein gel (Bio-Rad). After transferring the resolved proteins to a nitrocellulose membrane, membranes were blocked for $1.5 \mathrm{~h}$ in TBS-Tween $0.1 \%$ (TBS-T) $+5 \%$ blocking-grade blocker (Bio-Rad). Membranes were cut in two at the $37 \mathrm{kDa}$ marker position, and the lower and upper parts were incubated overnight at $4^{\circ} \mathrm{C}$ in TBS-T $+1 \%$ blocker and a 1:5,000 dilution of rabbit anti-human $\mathrm{Hb}$ antibody (Sigma \#H4890, lot 124M4788V) or a 1:2,000 dilution of $\beta$-actin monoclonal antibody (Fisher \#AM4302), respectively. After washing in TBS-T, membranes were incubated in TBS-T $+1 \%$ blocker and a 1:10,000 dilution of goat anti-rabbit IgG conjugated to horseradish peroxidase (GE Healthcare \#RPN4301) to detect $\mathrm{Hb}$, or a 1:10,000 dilution of sheep anti-mouse Ig coupled to horseradish 
peroxidase (GE Healthcare \#NXA931) to detect $\beta$-actin. Proteins bands were detected using the Pierce ECL western blotting substrate (ThermoScientific), blue basic autoradiography film (Genemate) and a Nikon X-OMAT 2000. Hb band intensities were calculated in ImageJ, normalized for loading with the $\beta$-actin signal, and compared to untreated controls. Experiments were repeated on three to four independent occasions.

Data availability. All data generated or analysed during this study are included in this published article and its Supplementary Information. Requests for materials should be addressed to the corresponding author.

Received: 18 July 2016; Accepted: 11 July 2017; Published online: 14 August 2017

\section{References}

1. World Malaria Report 2016 (WHO, 2016); http://www.who.int/malaria/ publications/world-malaria-report-2016

2. Wells, T. N. C., van Huijsduijnen, R. H. \& Van Voorhis, W. C. Malaria medicines: a glass half full? Nat. Rev. Drug Discov. 14, 424-442 (2015).

3. Sinden, R. E. Targeting the parasite to suppress malaria transmission. Adv. Parasitol. 97, 147-185 (2017).

4. Josling, G. A. \& Llinás, M. Sexual development in Plasmodium parasites: knowing when it's time to commit. Nat. Rev. Microbiol. 13, 573-587 (2015).

5. Bousema, T. \& Drakeley, C. Epidemiology and infectivity of Plasmodium falciparum and Plasmodium vivax gametocytes in relation to malaria control and elimination. Clin. Microbiol. Rev. 24, 377-410 (2011).

6. Cowman, A. F., Healer, J., Marapana, D. \& Marsh, K. Malaria: biology and disease. Cell 167, 610-624 (2016).

7. Dicko, A. et al. Primaquine to reduce transmission of Plasmodium falciparum malaria in Mali: a single-blind, dose-ranging, adaptive randomised phase 2 trial. Lancet Infect. Dis. 16, 674-684 (2016).

8. White, N. J. Primaquine to prevent transmission of falciparum malaria. Lancet Infect. Dis. 13, 175-181 (2013).

9. Tanaka, T. Q. et al. A quantitative high throughput assay for identifying gametocytocidal compounds. Mol. Biochem. Parasitol. 188, 20-25 (2013).

10. Cevenini, L. et al. Multicolor bioluminescence boosts malaria research: quantitative dual-color assay and single-cell imaging in Plasmodium falciparum parasites. Anal. Chem. 86, 8814-8821 (2014).

11. Sanders, N. G., Sullivan, D. J., Mlambo, G., Dimopoulos, G. \& Tripathi, A. K. Gametocytocidal screen identifies novel chemical classes with Plasmodium falciparum transmission blocking activity. PLoS ONE 9, e105817 (2014).

12. Bolscher, J. M. et al. A combination of new screening assays for prioritization of transmission-blocking antimalarials reveals distinct dynamics of marketed and experimental drugs. J. Antimicrob. Chemother. 70, 1357-1366 (2014).

13. Lucantoni, L., Duffy, S., Adjalley, S. H., Fidock, D. A. \& Avery, V. M. Identification of MMV malaria box inhibitors of Plasmodium falciparum early-stage gametocytes using a luciferase-based high-throughput assay. Antimicrob. Agents Chemother. 57, 6050-6062 (2013).

14. Sun, W. et al. Chemical signatures and new drug targets for gametocytocidal drug development. Sci. Rep. 4, 3743 (2014).

15. Bowman, J. D. et al. Antiapicoplast and gametocytocidal screening to identify the mechanisms of action of compounds within the malaria box. Antimicrob. Agents Chemother. 58, 811-819 (2014).

16. Plouffe, D. M. et al. High-throughput assay and discovery of small molecules that interrupt malaria transmission. Cell Host Microbe 19, 114-126 (2016).

17. Miguel-Blanco, C. et al. Imaging-based high-throughput screening assay to identify new molecules with transmission-blocking potential against Plasmodium falciparum female gamete formation. Antimicrob. Agents Chemother. 59, 3298-3305 (2015).

18. Lucantoni, L., Loganathan, S. \& Avery, V. M. The need to compare: assessing the level of agreement of three high-throughput assays against Plasmodium falciparum mature gametocytes. Sci. Rep. 7, 45992 (2017).

19. Delves, M. J. et al. Routine in vitro culture of $P$. falciparum gametocytes to evaluate novel transmission-blocking interventions. Nat. Protoc. 11, 1668-1680 (2016).

20. de Koning-Ward, T. F., Gilson, P. R. \& Crabb, B. S. Advances in molecular genetic systems in malaria. Nat. Rev. Microbiol. 13, 373-387 (2015).

21. Flannery, E. L., Fidock, D. A. \& Winzeler, E. A. Using genetic methods to define the targets of compounds with antimalarial activity. J. Med. Chem. 56, 7761-7771 (2013)

22. Plouffe, D. et al. In silico activity profiling reveals the mechanism of action of antimalarials discovered in a high-throughput screen. Proc. Natl Acad. Sci. USA 105, 9059-9064 (2008).

23. Adjalley, S. H. et al. Quantitative assessment of Plasmodium falciparum sexual development reveals potent transmission-blocking activity by methylene blue. Proc. Natl Acad. Sci. USA 108, E1214-E1223 (2011).
24. Coulibaly, B. et al. Efficacy and safety of triple combination therapy with artesunate-amodiaquine-methylene blue for falciparum malaria in children: a randomized controlled trial in Burkina Faso. J. Infect. Dis. 211, 689-697 (2015)

25. Fidock, D. A., Rosenthal, P. J., Croft, S. L., Brun, R. \& Nwaka, S. Antimalarial drug discovery: efficacy models for compound screening. Nat. Rev. Drug Discov. 3, 509-520 (2004).

26. Corey, V. C. et al. A broad analysis of resistance development in the malaria parasite. Nat. Commun. 7, 11901 (2016).

27. Ng, C. L. et al. CRISPR-Cas9-modified $p f m d r 1$ protects Plasmodium falciparum asexual blood stages and gametocytes against a class of piperazinecontaining compounds but potentiates artemisinin-based combination therapy partner drugs. Mol. Microbiol. 101, 381-393 (2016).

28. Ferreira, P. E. et al. PfMDR1: mechanisms of transport modulation by functional polymorphisms. PLoS ONE 6, 3-10 (2011).

29. Sidhu, A. B. S., Valderramos, S. G. \& Fidock, D. A. pfmdr1 mutations contribute to quinine resistance and enhance mefloquine and artemisinin sensitivity in Plasmodium falciparum. Mol. Microbiol. 57, 913-926 (2005).

30. Veiga, M. I. et al. Globally prevalent PfMDR1 mutations modulate Plasmodium falciparum susceptibility to artemisinin-based combination therapies. Nat. Commun. 7, 11553 (2016).

31. Sá, J. M. et al. Geographic patterns of Plasmodium falciparum drug resistance distinguished by differential responses to amodiaquine and chloroquine. Proc. Natl Acad. Sci. USA 106, 18883-18889 (2009).

32. Combrinck, J. M. et al. Optimization of a multi-well colorimetric assay to determine haem species in Plasmodium falciparum in the presence of anti-malarials. Malar. J. 14, 253 (2015).

33. Combrinck, J. M. et al. Insights into the role of heme in the mechanism of action of antimalarials. ACS Chem. Biol. 8, 133-137 (2013).

34. Fukami, T. \& Yokoi, T. The emerging role of human esterases. Drug Metab. Pharmacokinet. 27, 466-477 (2012).

35. Blagborough, A. M. et al. Transmission-blocking interventions eliminate malaria from laboratory populations. Nat. Commun. 4, 1812-1817 (2013).

36. De Mello, C. X. et al. Comparison of the sensitivity of imprint and scraping techniques in the diagnosis of American tegumentary leishmaniasis in a referral centre in Rio de Janeiro, Brazil. Parasitol. Res. 109, 927-933 (2011)

37. Gil, J. P. \& Krishna, S. pfmdr1 (Plasmodium falciparum multidrug drug resistance gene 1): a pivotal factor in malaria resistance to artemisinin combination therapies. Expert Rev. Anti. Infect. Ther. 15, 527-543 (2017).

38. Sigala, P. A. \& Goldberg, D. E. The peculiarities and paradoxes of Plasmodium heme metabolism. Annu. Rev. Microbiol. 68, 259-278 (2014).

39. Tilley, L., Straimer, J., Gnädig, N. F., Ralph, S. A. \& Fidock, D. A. Artemisinin action and resistance in Plasmodium falciparum. Trends Parasitol. 32, 682-696 (2016).

40. Venkatesan, M. et al. Polymorphisms in Plasmodium falciparum chloroquine resistance transporter and multidrug resistance 1 genes: parasite risk factors that affect treatment outcomes for $P$. falciparum malaria after artemetherlumefantrine and artesunate-amodiaquine. Am. J. Trop. Med. Hyg. 91, 833-843 (2014).

41. Petersen, I., Eastman, R. \& Lanzer, M. Drug-resistant malaria: molecular mechanisms and implications for public health. FEBS Lett. 585, 1551-1562 (2011)

42. Petersen, I. et al. Balancing drug resistance and growth rates via compensatory mutations in the Plasmodium falciparum chloroquine resistance transporter. Mol. Microbiol. 97, 381-395 (2015).

43. Roberts, L., Egan, T. J., Joiner, K. A. \& Hoppe, H. C. Differential effects of quinoline antimalarials on endocytosis in Plasmodium falciparum. Antimicrob. Agents Chemother. 52, 1840-1842 (2008).

44. Wunderlich, J., Rohrbach, P. \& Dalton, J. P. The malaria digestive vacuole. Front. Biosci. 1, 1424-1448 (2012).

45. Rohrbach, P. et al. Genetic linkage of $p f m d r 1$ with food vacuolar solute import in Plasmodium falciparum. EMBO J. 25, 3000-3011 (2006).

46. Reiling, S. J. \& Rohrbach, P. Monitoring PfMDR1 transport in Plasmodium falciparum. Malar. J. 14, 270 (2015).

47. Brunner, R. et al. UV-triggered affinity capture identifies interactions between the Plasmodium falciparum multidrug resistance protein 1 (PfMDR1) and antimalarial agents in live parasitized cells. J. Biol. Chem. 288, 22576-22583 (2013).

48. Pleeter, P., Lekostaj, J. K. \& Roepe, P. D. Purified Plasmodium falciparum multi-drug resistance protein (PfMDR 1) binds a high affinity chloroquine analogue. Mol. Biochem. Parasitol. 173, 158-161 (2010).

49. Cowman, A. F., Karcz, S., Galatis, D. \& Culvenor, J. G. A P-glycoprotein homologue of Plasmodium falciparum is localized on the digestive vacuole. J. Cell Biol. 113, 1033-1042 (1991).

50. Tumwebaze, $\mathrm{P}$. et al. Changing antimalarial drug resistance patterns identified by surveillance at three sites in Uganda. J. Infect. Dis. 215, 631-635 (2017)

51. Rottmann, M. et al. Spiroindolones, a potent compound class for the treatment of malaria. Science 329, 1175-1180 (2010). 
52. Sidhu, S. B. A., Uhlemann, A., Valderramos, S. G., Krishna, S. \& Fidock, D. A. Decreasing pfmdr1 copy number in Plasmodium falciparum malaria heightens susceptibility to mefloquine, lumefantrine, halofantrine, quinine and artemisinin. J. Infect. Dis. 194, 528-535 (2006).

53. ChEMBL-NTD (EMBL-EBI); https://www.ebi.ac.uk/chemblntd

54. Duffy, S. \& Avery, V. M. Identification of inhibitors of Plasmodium falciparum gametocyte development. Malar. J. 12, 408 (2013).

55. Lucantoni, L., Fidock, D. A. \& Avery, V. M. A luciferase-based, high-throughput assay for screening and profiling transmission-blocking compounds against Plasmodium falciparum gametocytes. Antimicrob. Agents Chemother. 60, 2097-2107 (2016).

56. Ruecker, A. et al. A male and female gametocyte functional viability assay to identify biologically relevant malaria transmission-blocking drugs. Antimicrob. Agents Chemother. 58, 7292-7304 (2014).

57. Duffy, S. \& Avery, V. M. Development and optimization of a novel 384-well anti-malarial imaging assay validated for high-throughput screening. Am. J. Trop. Med. Hyg. 86, 84-92 (2012).

58. Fletcher, S. \& Avery, V. M. A novel approach for the discovery of chemically diverse anti-malarial compounds targeting the Plasmodium falciparum coenzyme A synthesis pathway. Malar. J. 13, 343 (2014).

59. Delves, M. J. et al. Male and female Plasmodium falciparum mature gametocytes show different responses to antimalarial drugs. Antimicrob. Agents Chemother. 57, 3268-3274 (2013).

60. McNamara, C. W. et al. Targeting Plasmodium PI(4)K to eliminate malaria. Nature 504, 248-253 (2013).

61. Pereira, M. R. et al. In vivo and in vitro antimalarial properties of azithromycin-chloroquine combinations that include the resistance reversal agent amlodipine. Antimicrob. Agents Chemother. 55, 3115-3124 (2011).

62. Goodyer, I. D. \& Taraschi, T. F. Plasmodium falciparum: a simple, rapid method for detecting parasite clones in microtiter plates. Exp. Parasitol. 86, 158-160 (1997).

63. Manary, M. J. et al. Identification of pathogen genomic variants through an integrated pipeline. BMC Bioinformatics 15, 63-76 (2014).

64. Hameed, P. S. et al. Triaminopyrimidine is a fast-killing and long-acting antimalarial clinical candidate. Nat. Commun. 6, 6715 (2015).

65. Ekland, E. H., Schneider, J. \& Fidock, D. A. Identifying apicoplast-targeting antimalarials using high-throughput compatible approaches. FASEB J. 25, 3583-3593 (2011).

66. D'Alessandro, S. et al. A Plasmodium falciparum screening assay for anti-gametocyte drugs based on parasite lactate dehydrogenase detection. J. Antimicrob. Chemother. 68, 2048-2058 (2013).

67. Tsirigos, K. D., Peters, C., Shu, N., Lukas, K. \& Elofsson, A. The TOPCONS web server for consensus prediction of membrane protein topology and signal peptides. Nucleic Acids Res. 43, W401-407 (2015).

68. Alva, V. et al. The MPI Bioinformatics Toolkit as an integrative platform for advanced protein sequence and structure. Nucleic Acids Res. 44, W410-415 (2016)

69. Sali, A., Potterton, L., Yuan, F., Van Vlijmen, H. \& Karplus, M. Evaluation of comparative protein modeling by MODELLER. Proteins $\mathbf{2 3}$ 318-326 (1995).
70. Emsley, P. \& Lohkamp, B. Features and development of Coot. Acta Crystallogr. D D66, 486-501 (2010).

71. Krieger, E. \& Vriend, G. Models @ Home: distributed computing in bioinformatics using a screensaver based approach. Bioinformatics 18, 315-318 (2002).

72. Bikadi, Z. \& Hazai, E. Application of the PM6 semi-empirical method to modeling proteins enhances docking accuracy of AutoDock. J. Cheminform 1, 15 (2009)

\section{Acknowledgements}

The authors thank T.T. Diagana (Novartis Institute for Tropical Diseases, Singapore) for provision of the compounds, the Red Cross (Australia and the USA) for the provision of human blood for cell cultures, and G. Stevenson for assistance with the triaging of compounds following screening. The authors acknowledge the Bill and Melinda Gates Foundation (grant OPP1040399 to D.A.F. and V.M.A. and grant OPP1054480 to E.A.W. and D.A.F.), the National Institutes of Health (grant R01 AI103058 to E.A.W. and D.A.F., grant R01 AI50234 to D.A.F, and R01 AI110329 to T.J.E.), the Australian Research Council (LP120200557 to V.M.A.) and the Medicines for Malaria Venture for their continued support. P.E.F. and M.I.V. are supported by the Northern Portugal Regional Operational Programme (NORTE 2020), under the Portugal 2020 Partnership Agreement, through the European Regional Development Fund (FEDER).

\section{Author contributions}

L.L. and S.D. screened the Novartis-GNF Malaria Box against early- and late-stage GAMs using LUC or GFP-imaging technologies, assayed ABS parasites and mammalian cells and confirmed compound potency. L.L., S.D. and V.M.A. analysed screening data and selected the HHQs for further evaluation. T.L., K.L.S. and S.L.H. contributed in vitro membrane feeding and male gametocyte exflagellation assay data. A.R., R.E.S. and M.D. contributed the DGFA data. M.V. and T.R.S.K. performed in vivo efficacy studies. K.R., M.V. and T.R.S.K. performed in vivo transmission-blocking studies. S.G. performed selections for HHQ-resistant lines, which were cloned by O.L. and M.V. Whole-genome sequence analysis was performed by V.C.C., P.P.H. and E.A.W. Gene editing of $p f m d r 1$ was performed by M.V. with help from C.L.N. and D.A.F. Susceptibility assays on resistant lines with clinical and experimental antimalarials were performed by M.V. with help from J.M.M. Early- and late-stage GAM susceptibility testing was performed by G.S. and P.A. Haem fractionation data were provided by J.M.C. and T.J.E. PfMDR1 modelling studies were performed by P.E.F and M.I.V. Data were compiled by M.V., L.L. and D.A.F., who wrote the manuscript with input from V.M.A. All authors approved of the final version.

\section{Competing interests}

The authors declare no competing financial interests.

\section{Additional information}

Supplementary information is available for this paper at doi:10.1038/s41564-017-0007-4. Reprints and permissions information is available at www.nature.com/reprints. Correspondence and requests for materials should be addressed to D.A.F. Publisher's note: Springer Nature remains neutral with regard to jurisdictional claims in published maps and institutional affiliations. 


\section{natureresearch}

\section{Life Sciences Reporting Summary}

Nature Research wishes to improve the reproducibility of the work we publish. This form is published with all life science papers and is intended to promote consistency and transparency in reporting. All life sciences submissions use this form; while some list items might not apply to an individual manuscript, all fields must be completed for clarity.

For further information on the points included in this form, see Reporting Life Sciences Research. For further information on Nature Research policies, including our data availability policy, see Authors \& Referees and the Editorial Policy Checklist.

\section{- Experimental design}

1. Sample size

Describe how sample size was determined.

The in vitro drug assays described in this manuscript were performed 3-12 times. This number of independent repeats exceeds standard number of 2-3 described in most studies. A minimum of 3 independent repeats is required to assess for a significant shift. The in vivo efficacy and transmission blocking data were performed on 2 different occasions using 4 mice per group, per standard protocols and to minimize the number of animals under experiment. Details regarding sample size of these and all other performed experiments are provided in the Methods section, the legends of the figures and in the supplementary files.

\section{Data exclusions}

Describe any data exclusions.

All data generated during this study are shown in the manuscript.

3. Replication

Describe whether the experimental findings were reliably reproduced.

All attempts at replication were successful. Data are represented as means with the corresponding standard error of the mean to indicate biological variation.

4. Randomization

Describe how samples/organisms/participants were allocated into experimental groups.

Outbred CD1 mice and mosquitoes were randomly allocated into treated and control groups for the in vivo efficacy and transmission-blocking experiments.

\section{Blinding}

Describe whether the investigators were blinded to group allocation during data collection and/or analysis.

Groups of mice ( 3 treated groups vs 1 untreated group) were blinded for collection and analysis of data when used for in vivo drug efficacy and feeding to mosquitoes for transmission-blocking studies. 


\section{Statistical parameters}

For all figures and tables that use statistical methods, confirm that the following items are present in relevant figure legends (or the Methods section if additional space is needed).

n/a $\mid$ Confirmed

The exact sample size $(n)$ for each experimental group/condition, given as a discrete number and unit of measurement (animals, litters, cultures, etc.)

A description of how samples were collected, noting whether measurements were taken from distinct samples or whether the same sample was measured repeatedly.

$\bigotimes$ A statement indicating how many times each experiment was replicated

The statistical test(s) used and whether they are one- or two-sided (note: only common tests should be described solely by name; more complex techniques should be described in the Methods section)

$\bigotimes$ A description of any assumptions or corrections, such as an adjustment for multiple comparisons

\The test results (e.g. $p$ values) given as exact values whenever possible and with confidence intervals noted

$\bigotimes$ A summary of the descriptive statistics, including central tendency (e.g. median, mean) and variation (e.g. standard deviation, interquartile range)

Х Clearly defined error bars

See the web collection on statistics for biologists for further resources and guidance.

\section{- Software}

Policy information about availability of computer code

\section{Software}

Describe the software used to analyze the data in this study.

Drug susceptibility data generation and statistical analysis were performed using GraphPad Prism 6 as mentioned in the respective paragraphs in the Methods section. Whole-genome sequencing analysis were performed using existing published tools as described in the manuscript (lines 692-694)

For all studies, we encourage code deposition in a community repository (e.g. GitHub). Authors must make computer code available to editors and reviewers upon request. The Nature Methods guidance for providing algorithms and software for publication may be useful for any submission.

\section{- Materials and reagents}

Policy information about availability of materials

\section{Materials availability}

Indicate whether there are restrictions on availability of unique materials or if these materials are only available for distribution by a for-profit company.

\section{Antibodies}

Describe the antibodies used and how they were validated for use in the system under study (i.e. assay and species).

There are no restrictions on availability of parasite strains or hexahydroquinoline compounds used in this study.

All antibodies and their origin were purchased at official suppliers as listed in the manuscript on lines 764-769 and are summarized below

- anti-human polyclonal hemoglobin antibody produced in rabbit (Sigma \#H4890, lot 124M4788V)

- goat anti-rabbit IgG horseradish peroxidase (GE Healthcare \#RPN4301)

- anti-mouse beta-actin monoclonal antibody (AC-15) (Fisher \#AM4302)

- sheep anti-mouse Ig, horseradish peroxidase linked whole antibody (GE Healthcare \#NXA931) 
10. Eukaryotic cell lines

a. State the source of each eukaryotic cell line used.

All parasite lines that were not generated in this study are described on lines 534-539

b. Describe the method of cell line authentication used.

Cell lines were verified by PCR and Sanger sequencing of different loci known to differ between parasite lines (such as PfMDR1 for example (see line 712-713)).

c. Report whether the cell lines were tested for mycoplasma

Original cell lines were tested by PCR for Mycoplasma contamination every contamination.

2 to 3 months (see line 539).

d. If any of the cell lines used in the paper are listed in the database

No commonly misidentified cell lines were used.

of commonly misidentified cell lines maintained by ICLAC, provide a scientific rationale for their use.

\section{- Animals and human research participants}

Policy information about studies involving animals; when reporting animal research, follow the ARRIVE guidelines

\section{Description of research animals}

Provide details on animals and/or animal-derived materials used in the study.

Policy information about studies involving human research participants

\section{Description of human research participants}

Describe the covariate-relevant population characteristics of the human research participants.
Animals used for the in vivo studies: Mus musculus, CD-1 strain, female, 22-24 grams (as mentioned on lines 653-654).

The study did not involve human research participants. 